\title{
CONSTRUCTIVE VERSIONS OF ORDINAL NUMBER CLASSES
}

\author{
BY \\ DONALD L. KREIDER AND HARTLEY ROGERS, JR.(1)
}

0. Introduction and summary. Almost from its beginning, the theory of ordinal numbers has included results which can, in a rather concrete sense, be viewed as theorems about notations for ordinal numbers. For example the theorems on polynomial normal forms for those ordinals that lie below the least $\epsilon$ number can be understood as providing a certain unique notation of finite length for each such ordinal (where the notation involves addition, multiplication and exponentiation applied to the integers and to $\omega$ ) [B]. Indeed, if we consider notations for some but not all ordinals in a given segment, then a large part of the theory of ordinals can be viewed as concerned with notations. If we limit our attention to those classical results which directly provide systems of notation for segments of the countable ordinals (e.g. the polynomial theorems mentioned above), we note the following common features:

(i) given any notation for a successor ordinal, we can effectively find a notation for its predecessor;

(ii) given any notation for a limit ordinal, we can effectively find notations for ordinals in a fundamental sequence to the given limit ordinal. (A fundamental sequence to ordinal $\alpha$ is a strictly monotone sequence whose limit is $\alpha$.)

The notational aspects of ordinal number theory remained unclear for some time. Using the tools of recursive function theory, Church and Kleene first satisfactorily resolved this unclarity in $[\mathrm{C}]$, in $[\mathrm{CK}]$ and in $\left[\mathrm{K}_{1}\right]$. By adopting the integers themselves as a standard infinite collection of available labels $\left({ }^{2}\right)$, and by interpreting (i) and (ii) above in terms of recursive computability, it is possible to characterize precisely the concept of an effective system of notation, (the " $r$-systems" of $\left.\left[\mathrm{K}_{1}\right]\right)$. Furthermore, by sacrificing recursiveness of the set of all notations of such a system, one is able to define effective systems which are maximal in the sense that they cover a segment of ordinals as large as is covered by any effective system. The ordinals covered by such

Received by the editors September 27, 1960.

(1) Many of the results presented below were first obtained in the doctoral dissertation of Kreider [Kr]. Work of both authors was supported in part by a project sponsored by the Air Force Office of Scientific Research of the Air Research and Development Command under contract AF 49(638-76).

(2) Note that we are using integers (set theoretic objects) rather than numerals (syntactical objects) as labels. Although the use of numerals would be closer to our motivation, the use of integers simplifies statements of theorems and proofs. For our purposes the distinction is of minor importance. 
a maximal system are called the constructive ordinals and form a proper segment of the countable ordinals. By allowing ordinals to have more than one notation, it is possible to define maximal systems into which any effective system can be mapped (indeed, effectively mapped) in an order-preserving way.

Two such maximal systems have been of especial interest: the system of Church and Kleene (called $S_{1}$ in $\left[K_{1}\right]\left({ }^{3}\right)$ ); and the system of Kleene (called $S_{3}$ in $\left[\mathrm{K}_{1}\right]$, usually associated with the symbols $O$ and $<_{0}$ in later literature). The system $S_{3}$ has been more widely used in subsequent applications, owing to advantages to be mentioned below (cf. §2); although the system $S_{1}$ has the advantage that any system can be mapped into it in an ordinal-preserving (and not just order-preserving) way. These systems have had three general applications.

(1) They have given direct insight into certain "notational" (and quasiphilosophical) aspects of ordinal number theory.

(2) They have provided (denumerable) "constructive analogues" to the (nondenumerable) segment of all countable ordinals. Each of the two systems $S_{1}$ and $S_{3}$ is such an analogue in the sense that its notations are closed under certain recursive rules analogous to (noneffective) closure rules on ordinals that are known to determine the segment of countable ordinals.

(3) They supply, in their structure, a natural and useful method for iterating effective procedures out into the transfinite.

Although their original definition is noninvariant, the systems have, in application (3), led to recursively invariant structures of considerable interest. For example, effective iteration using the jump operation on sets (cf. $\left[\mathrm{K}_{3}\right]$ ) leads to the hyperarithmetical sets.

In the present paper we are concerned with extending the systems $S_{1}$ and $S_{3}$ to cover a larger segment of ordinals. We do so at the expense of some of their effective structure. (We will weaken condition (ii).) These extensions will be made in a way that parallels the generation of higher "number classes" in the classical theory of ordinals. In terms of this parallel, the extension will be carried as far as the first inaccessible ordinal. Our new systems will be useful for essentially the same reasons as before. In particular they will:

(1) give insight into the "notational" aspect of accessibility;

(2) give a proper segment of the countable ordinals which is a direct analogue to the segment of ordinals out to the least inaccessible ordinal;

(3) provide a means for more extensive iteration of certain effective processes.

In [AK], Addison and Kleene define an extension of $S_{3}$ that is analogous

(3) $S_{1}$ uses integers as notations, whereas the original Church-Kleene system uses formal expressions of a lambda calculus. In its recursive structure, the latter is isomorphic to $S_{1}$ in all respects. 
to the segment of ordinals through the "third number class" (i.e. through ordinals of cardinality $\boldsymbol{N}_{1}$ ); and they show that iteration of the hyperjump over this extension does not lead beyond sets in both two-function-quantifier forms. We extend this result to our systems, thus settling a question suggested by Kleene to one of the authors.

More specifically, we shall proceed as follows. In $\$ 1$ we give certain terminology and background related to the classical theory of ordinals. In $\$ 2$ we discuss $S_{1}, S_{3}$ and the general notion of system of notations. In $\$ 3$ we discuss certain peculiarities of the Addison-Kleene extension of $S_{3}$. In $\$ 4$ we extend $S_{1}$. In $\$ 5$ we extend $S_{3}$ (in continuation of the Addison-Kleene extension). In $\$ 6$ we show that the system of $\$ 4$ is in both two-function-quantifier forms. In $\$ 7$ we use this result to show that iteration of the hyperjump over the extension of $S_{1}$ does not lead beyond sets in both two-function-quantifier forms. In $\$ 8$ we obtain the results of $\$ \$ 6-7$ for the extension of $S_{3}$. In $\$ 9$ we raise certain open questions and discuss possible directions for further research.

1. Ordinal number classes. We assume a standard axiomatization of set theory, e.g. that of $[G]$. Notation: $\alpha, \beta, \gamma, \cdots$ for ordinals; $\omega$ for the least infinite ordinal; $|\alpha|$ for the cardinal number of $\alpha$. We give certain definitions and results from the theory of ordinals. In order to motivate our subsequent constructions, we use the notion of type number (sometimes called regular number, see $[\mathrm{F}])$. Proofs are routine and are omitted.

Definition. $\alpha$ is an initial number $\equiv_{d f}(\forall \beta)[|\beta|=|\alpha| \Rightarrow \beta \geqq \alpha]$.

Let $A$ and $B$ be classes of ordinals, then we have:

Definition. $A$ and $B$ are cofinal $\equiv_{d f}$

$$
\begin{aligned}
(\forall \alpha)[\alpha \in A & \Rightarrow(\exists \beta)[\beta \in B \& \beta \geqq \alpha]] \\
\&(\forall \beta)[\beta \in B & \Rightarrow(\exists \alpha)[\alpha \in A \& \alpha \geqq \beta]] .
\end{aligned}
$$

REsult 1. If $A$ is cofinal with the class of all ordinals, then $A$ is orderisomorphic to the class of all ordinals.

RESULT 2. The class of infinite initial numbers is cofinal with the class of all ordinals.

It follows that the class of infinite initial numbers is order-isomorphic to the class of all ordinals. Let $f$ be the isomorphism function mapping the class of all ordinals onto the infinite initial numbers. $f$ provides an indexing of the infinite initial numbers.

Definition. $\omega_{\alpha}=d_{j} f(\alpha)$.

RESULT 3. $f$ is monotone and continuous (in the usual interval topology). Hence by the theorem of Veblen in [V] it has a fixed point. The least such fixed point is the limit of the sequence $\omega, \omega_{\omega}, \omega_{\omega_{\omega}}, \cdots$, which is sometimes written $\omega_{\omega_{\omega} \ldots . . .}$ (This number is accessible in the sense to be defined below.)

Definition. If $g$ is an order-preserving map from the predecessors of $\beta$ into the ordinals, we shall sometimes denote $g$ by $\left\{\alpha_{\iota}\right\}_{\iota<\beta}$ where $\alpha_{\imath}=d_{d f} g(\iota)$.

RESULT 4. It follows from the first result above that given any $\left\{\alpha_{\imath}\right\}_{\llcorner<\beta}$ then $\left\{\gamma \mid \gamma>\alpha_{\iota}\right.$ for all $\left.\iota<\beta\right\}$ is not empty. 
Definition. Let $\left\{\alpha_{\imath}\right\}_{c<\beta}$ be given, then

$$
\begin{aligned}
& \lim _{\iota<\beta} \alpha_{\imath}=d_{d f} \text { least member of }\left\{\gamma \mid \gamma>\alpha_{\imath} \text { for all } \iota<\beta\right\} . \\
& \alpha \text { is of limit type } \beta \equiv_{d f} \alpha=\lim _{\iota<\beta} \alpha_{\imath} \text { for some }\left\{\alpha_{\iota}\right\}_{\iota<\beta} .
\end{aligned}
$$

( $\beta$ is sometimes called a final character of $\alpha$.) $\boldsymbol{\gamma}]$.

$\alpha$ is of similar type to $\beta \equiv_{d f}(\exists \gamma)[\alpha$ is of limit type $\gamma$ and $\beta$ is of limit type

RESULT 5. The relation " $\alpha$ is of similar type to $\beta$ " is reflexive, transitive and symmetric.

Definition. $\alpha$ is a type number $\equiv_{d f}(\forall \beta)[\beta$ of similar type to $\alpha \Rightarrow \beta \geqq \alpha]$. (Note the analogy between " $\beta$ of similar type to $\alpha$ " and " $|\beta|=|\alpha|$ "; and hence between "type number" and "initial number." The type numbers are just those numbers determining segments which could replace the class of all ordinals in Result 1 above. I.e., $\alpha$ is a type number if and only if, for all classes $A, A$ cofinal with the predecessors of $\alpha \Rightarrow A$ of order type $\alpha$. There are two finite type numbers; 0 and $1 . \omega$ is the least infinite type number.)

The following results have routine but not uninteresting proofs.

REsults 6. (a) The least limit type of an ordinal is a type number.

(b) Every type number is an initial number.

(c) If $\alpha$ is a successor number, (i.e. if it has a greatest predecessor), then $\omega_{\alpha}$ is a type number.

(d) Not all infinite initial numbers are type numbers (e.g. $\omega_{\omega}$ is of type $\left.\omega\right)$; however, for any $\alpha$, the least type number exceeding $\alpha$ is equal to the least initial number exceeding $\alpha$.

(e) The class of infinite type numbers is cofinal with the class of all ordinals.

Hence, as with the initial numbers, we obtain an isomorphism and a function $h$ that gives an indexing of the infinite type numbers.

Definition. $\tau_{\alpha}={ }_{d f} h(\alpha)$. This function is not continuous, (e.g. at $\omega$ ), and hence we cannot apply the Veblen theorem to obtain a fixed point. The fixed points of this function are just the inaccessible ordinals. Whether or not the existence of such ordinals can be proved depends (more sensitively than any of the above) on the particular axiomatization of set theory being used. Regardless of existence, the following theorem can be proved.

RESUlT 7. $\tau_{\alpha}=\alpha \Leftrightarrow\left[\omega_{\alpha}=\alpha\right.$ and $\alpha$ is not of limit type less than $\alpha[\Leftrightarrow] \omega_{\alpha}=\alpha$ and $\alpha$ is a type number].

Hence we have the more conventional definition:

Definition. $\alpha$ is inaccessible $\equiv_{d f} \omega_{\alpha}=\alpha$ and $\alpha$ is not of limit type less than $\alpha$.

The relationship between type numbers and initial numbers is completed by:

RESULT 8. (a) If $\alpha$ is not a successor number, then $\omega_{\alpha}$ is a type number if and only if $\alpha=0$ or $\alpha$ is inaccessible. 
(b) Hence $\tau_{\alpha}=\omega_{\alpha}$ if there is $a \delta=0$ or $\delta$ inaccessible such that $\delta \leqq \alpha<\delta+\omega$; otherwise $\tau_{\alpha}=\omega_{\alpha+1}$.

The following terminology is traditional.

DEFINITION.

$$
\aleph_{\alpha}={ }_{d f}\left|\omega_{\alpha}\right| \text {. }
$$

The first Number Class $=_{d f}\left\{\boldsymbol{\gamma}|| \boldsymbol{\gamma} \mid<\aleph_{0}\right\}$.

For $2 \leqq \alpha<\omega$, the $\alpha$ th Number Class $=_{d f}\left\{\gamma|| \gamma \mid=\boldsymbol{\aleph}_{\alpha-2}\right\}$.

For $\omega \leqq \alpha$, the $\alpha$ th Number Class ${ }_{d f}\left\{\gamma|| \gamma \mid=\aleph_{\alpha}\right\}$.

Let $N_{\beta}$ be the $\beta$ th Number Class.

Definition. The $\alpha$ th cumulative number class $=_{d f} \bigcup_{\beta \leq \alpha} N_{\beta}$. We shall find it convenient to use cumulative number classes. Henceforth the phrase "number class" will mean "cumulative number class" $\left.{ }^{4}\right)$. The following characterization of number classes is immediate.

RESULT 9. For $1 \leqq \alpha<\omega$, the $\alpha$ th number class $=\left\{\gamma \mid \gamma<\tau_{\alpha-1}\right\}$.

For $\omega \leqq \alpha<$ the least inaccessible ordinal, the $\alpha$ th number class $=\left\{\gamma \mid \gamma<\tau_{\alpha}\right\}$. (Note that, although $\omega_{\omega} \ldots$ is accessible, the first number class in which $\omega_{\omega} \ldots$ appears is the number class of index $\omega_{\omega} \ldots$.)

This result suggests that the ordinals be viewed as obtained in sequence from preceding ordinals by certain principles of generation (or closure rules). In particular, consider the principles:

(i) include 0 and 1 as ordinals;

(ii) given any $\left\{\alpha_{1}\right\}_{<<\beta}$, where $\beta$ is a type number and where $\beta$ and all the $\alpha_{\imath}$ have already been obtained, include $\lim _{\iota<\beta} \alpha_{\iota}$ as an ordinal;

(iii) at any "point of difficulty" where both (i) and (ii) fail to apply, introduce the next ordinal and label it has the $\alpha$ th point of difficulty providing $\alpha$ has already been obtained and $\alpha$ is the least ordinal not previously used to label a point of difficulty. (Note that (ii) is equivalent to the apparently more general rule: given any $\left\{\alpha_{\imath}\right\}_{\iota<\beta}$, where $\beta$ and all the $\alpha_{\iota}$ have already been obtained, include $\lim _{\mathrm{\iota}<\beta} \alpha_{\mathrm{\imath}}$.)

These principles, or closure rules, give us exactly the ordinals out to the least inaccessible ordinal. The "points of difficulty" are the infinite type numbers, with $\tau_{\alpha}$ as the $\alpha$ th point of difficulty. Note that if we allow only one application of (iii), to introduce $\tau_{0}=\omega$, then we obtain exactly the 2nd number class. (Here, applications of (ii) give only ordinals of limit type 1 or limit type $\omega$.) A second application of (iii) would give the third number class, etc. As we shall see, our extensions of systems of notations will parallel these clossure rules for "generating" the classical ordinals.

In conclusion we note a final theorem about type numbers.

RESULT 10. $\alpha$ of limit type $\tau_{\gamma}$ and $\alpha$ of limit type $\tau_{\delta} \Rightarrow \gamma=\delta$.

(Hence we could speak of $\tau_{\gamma}$ as the type of $\alpha$.) Most of the results above have true analogues for our constructive systems. However, the truth of an

(4) We shall sometimes refer to the $\alpha$ th number class as "the number class of index $\alpha$ ". 
analogue to this last result remains an open question.

Final Comment. The reader will be able to conceive of further more powerful principles of generation that may be added to (i)-(iii) above. Thus he might add:

(iv) At any "point of 2nd order difficulty", where (i)-(iii) fail to apply, introduce the next ordinal and label it the $\alpha$ th point of 2 nd order difficulty providing $\alpha$ has already been obtained and $\alpha$ is the least ordinal not previously used to label a point of 2nd order difficulty.

He might further add rules to cover points of $n$th order difficulty, or, indeed, points of $\beta$ th order difficulty where $\beta$ is any ordinal already obtained.

He can now have "points of super difficulty" where all the preceding fail to apply, "points of $\gamma$ th order super difficulty", etc., etc.

The existence of such (generalized) points of difficulty will depend upon the axiomatization for set theory being used. (Indeed, this provides an interesting measure of the strength of a set theory.) Assuming, by axiom, the existence of more and more general points of difficulty is one natural way of strengthening the usual set theories. Study of this is begun in [L]. As we shall observe below, the theory of effective and quasi-effective systems of notation may be a useful tool in such studies.

2. Systems of notation; $S_{1}$ and $S_{3}$.

Definition. $R$ is a partial ordering $\equiv_{d f} R$ is a transitive and irreflexive binary relation.

For partial ordering $R, x<_{R} y \equiv_{d f}\langle x, y\rangle \in R$.

$$
\text { domain of } R \text { (“dom } R \text { ") }{ }_{d f}\{x \mid(\exists y)[\langle x, y\rangle \in R \text { or }\langle y, x\rangle \in R]\} \text {. }
$$

$R$ is a well-ordered partial ordering $\equiv_{d f} R$ is a partial ordering and every nonempty subset of $d o m R$ has a minimal element.

For $R$ a partial ordering of integers, it is easy to show (without the axiom of choice) that $R$ is well-ordered if and only if $R$ satisfies the descending chain condition (i.e. it has no infinite descending chains). (Well-ordered partial orderings are sometimes called well-founded relations.)

For reasons that will appear, it seems appropriate to identify the general concept of a system of notations for ordinals with that of a well-ordered partial ordering of integers. Let $N$ be the set of non-negative integers.

DEFINITION. $R$ is a system of notations $\equiv_{d f} R$ is a well-ordered partial ordering and $R \subseteq N \times N$.

A chief justification for this definition is found in the following theorem.

TheOREM 1. Let $R$ be a system of notations. There is a natural order-preserving map from dom $R$ onto a segment of the second number class.

Proof. The map, call it $f$, is defined inductively by:

$$
f(x)=\text { least member of }\left\{\gamma \mid(\forall y)\left[y<_{R} x \Rightarrow f(y)<\gamma\right]\right\} .
$$


It is easy to show existence and uniqueness of such an $f$ and that $x<_{R} y \Rightarrow f(x)$ $<f(y)$. The map $f$ is natural inasmuch as, for any other order-preserving map $g$,

$$
f(x) \leqq g(x) \text { for all } x \text { in } \operatorname{dom} R .
$$

Comment. Our restriction to integers may appear to rule out certain traditional systems of notation. This is not the case, however, since we are developing our theory within the framework of recursive invariance. The notations of any such traditional system can be effectively mapped one-one ("encoded by Gödel numbers") onto some set of integers.

As a simple example of a system of notations, consider the linear ordering: $1,2,2^{2}, 2^{2}, \cdots$. Under the mapping of Theorem 1 , this system provides a notation for each finite ordinal: 1 is a notation for the ordinal 0 , and if $x$ is a notation for finite ordinal $n$, then $2^{x}$ is a notation for $n+1$. We use the following abbreviation for the inverse mapping to $f$ in this case.

Definition. $\overline{0}={ }_{d f} 1$,

$$
\overline{n+1}={ }_{d f} 2^{n} .
$$

Thus $\bar{n}$ is the notation for ordinal $n$. (Kleene uses the symbol " $n_{o}$ " for our " $\bar{n}^{\eta}$.) This particular system of notations for the finite ordinals will be a part of each of the specific larger systems to be considered below.

We next turn to the system $S_{1}$. This is given in $\left[K_{1}\right]$ by an "inductive definition" which simultaneously defines a set of notations and a mapping from notations into the ordinals. Let $\phi_{a}$ be the partial recursive function with Gödel number $e$. We use " $S$ " to denote the set of notations and " $\mid s$ " to denote the mapping from notations into ordinals. The definition runs as follows:

(a) $1 \in S \&|1|_{s=0}$

(b) $x \in S \Rightarrow\left[2^{x} \in S \&\left|2^{x}\right|_{S}=|x|_{s}+1\right]$;

(c) $\left[(\forall x)\left[\phi_{e}(\bar{x})\right.\right.$ defined and in $\left.S\right] \&\left\{\left|\phi_{\theta}(\bar{x})\right|_{S}\right\}_{x=0}^{x=\infty}$ an increasing sequence of ordinals $] \Rightarrow\left[3.5^{e} \in S \&\left|3 \cdot 5^{e}\right|_{S}=\lim _{x}\left|\phi_{\theta}(\bar{x})\right|_{S}\right]$.

Basic properties of $S_{1}$ are developed in [ $\left.\mathrm{K}_{1}\right]$. The question naturally arises: can this be formulated as a system in our sense? If we make the definition

$$
x<_{S} y \equiv_{d f} x \in S \& y \in S \&|x|_{S}<|y|_{s},
$$

we obtain a partial ordering; and both the set $S$ and the mapping ||$s$ can be recovered from $<_{s}$ by taking $S$ as $\operatorname{dom}\left(<_{S}\right)$ and ||$_{s}$ as the mapping $f$ of Theorem 1 . Hence $<_{S}$ does give the system $S_{1}$ in our sense. The reader should note the analogy between the definition of $S_{1}$ and the definition of the second number class by closure rules as suggested before Result 10 in $\$ 1$. The three 
conditions for $S_{1}$ correspond to inclusion of 0 , of ordinals of limit type 1 (successors), and of ordinals of limit type $\omega$.

The system $S_{3}$ is also given in $\left[\mathrm{K}_{1}\right]$ by an "inductive definition" which, in this case, simultaneously defines a set of notations, a mapping from notations into ordinals, and a partial ordering. Following Kleene, we use $O$ for the set, || for the mapping, and $<_{o}$ for the ordering. The definition runs as follows:

(a) $1 \in O \&|1|=0$;

(b) $x \in O \Rightarrow\left[2^{x} \in O \& x<<_{0} 2^{x} \&\left|2^{x}\right|=|x|+1\right]$;

(c) $\left[x<_{0} y \& y<_{0} z\right] \Rightarrow x<0 z$

(d) $\left[(\forall x)\left[\phi_{e}(\bar{x})\right.\right.$ defined and in $\left.\left.O\right] \&(\forall x, y)\left[x<y \Rightarrow \phi_{e}(\bar{x})<_{o} \phi_{e}(\bar{y})\right]\right]$ $\Rightarrow\left[3 \cdot 5^{e} \in O \&(\forall x)\left[\phi_{e}(\bar{x})<_{o} 3 \cdot 5^{e}\right] \&\left|3 \cdot 5^{e}\right|=\lim _{x}\left|\phi_{e}(\bar{x})\right|\right]$.

Basic properties of $S_{3}$ are developed in [K $\mathrm{K}_{1}$. Both $S_{1}$ and $S_{3}$ assign notations to the same segment of ordinals (the constructive ordinals). We first observe that the relation $<_{o}$ gives $S_{3}$ as a system in our sense; $O$ and $\mid$ can be recovered from it, as before with $S_{1}$. We also note that mention of || can be deleted from the inductive conditions without affecting $O$ and $<_{o}$, since, as Kleene pointed out, it does not occur on the left hand side of any condition. The $S_{3}$ definition differs from the $S_{1}$ definition, of course, in that the left hand side of the last condition for $S_{3}$ is more restrictive. The system $S_{3}$ is contained in the system $S_{1}$ (either as relation, as set, or as mapping). The system $S_{3}$ has been used in applications almost exclusively, since it has the convenient property that the set of predecessors of any $x$ (w.r.t. $<_{0}$ ) is linearly ordered and uniformly recursively enumerable in $x$.

It would be interesting if the system $S_{1}$ could also be defined without reference to ||$s$, the mapping into the ordinals. (We would then have what Kleene calls a "number-theoretic" definition of $S_{1}$.) It would also be interesting if the somewhat loosely presented "inductive definitions" above could be replaced by more basic and conventional "explicit" set theoretic constructions requiring no reference to ordinal structure. We next present such constructions in summary form. As they will be part of the more general constructions of $\S 4$ and $\S 5$, appropriate proofs and justifications will be postponed until then.

We give first the definition of $S_{3}$, i.e. of $<_{o}$. We use the following temporary definition:

$R$ is admissible $\equiv_{d f}(\mathrm{i})\langle 1,1\rangle \in R$,

(ii) $\langle 1, x\rangle \in R \Rightarrow\left\langle x, 2^{x}\right\rangle \in R$,

(iii) $\langle x, y\rangle \in R \&\langle y, z\rangle \in R \Rightarrow\langle x, z\rangle \in R$,

(iv) $\left[(\forall x)\left[\phi_{e}(\bar{x})\right.\right.$ defined $\left.] \&(\forall x, y)\left[x<y \Rightarrow\left\langle\phi_{e}(\bar{x}), \phi_{e}(\bar{y})\right\rangle \in R\right]\right]$ $\Rightarrow(\forall x)\left[\left\langle\phi_{e}(\bar{x}), 3 \cdot 5^{e}\right\rangle \in R\right]$.

Let $a$ be the collection of all admissible relations. $a$ is nonempty since $N \times N \in$ a. Let

$$
\hat{R}={ }_{d f} \bigcap_{R \in Q} R
$$


THEOREM $2\left({ }^{5}\right) . \hat{R}=<0$.

Proof. It follows from the definition of $\hat{R}$ that $\hat{R}$ is itself admissible, and by use of this fact it is possible to show that $\hat{R}$ is a system of notations, that $\hat{R}$ and dom $\hat{R}$ satisfy the conditions of the first definition for $<_{o}$ and $O$, and that this is true of no proper subrelation of $\hat{R}$. The general technique of proof is to show that if $\hat{R}$ failed to have any desired property, then $\hat{R}$ would possess an admissible proper subrelation, contradicting the definition of $\hat{R}$. (See §5.) Q.E.D.

The definition of $S_{1}$ is somewhat more complex. Let $\Re$ be the collection of all subsets of $N \times N$. We first define an operation, denoted by $f^{*}$, which maps $\Re$ into $\Re$. It will have the property that $R \subseteq f^{*}(R)$ for any $R \in \mathfrak{T}$. [Though we shall not use ordinals in our definition, we make the following comment to supply motivation. Let $\lesssim$ be the relation defined by $x \lesssim y$ $\equiv_{d f} x \in S \& y \in S \&|x|_{s} \leqq|y| s .<_{s}$ can be obtained from $\lesssim$ by the equivalence $x<_{s} y \Leftrightarrow[x \lesssim y \&$ not $y \lesssim x]$. If $\beta$ is a constructive ordinal and if $R$ is the relation $\lesssim$ restricted to notations for $\{\gamma \mid \gamma<\beta\}$, then $f^{*}(R)$ will be the relation $\lesssim$ restricted to notations for $\{\gamma \mid \gamma \leqq \beta\}$. Our construction amounts to making iterated applications of $f^{*}$ until all of $\lesssim$ is generated, and then defining $<$ from $\lesssim$.]

We make the following preliminary definitions; these definitions apply to any relation $R$, although the terminology is appropriate only to those relations "generated" from $\{\langle 1,1\rangle\}$ by $f^{*}$. $\in R]$.

Definition. $x$ is maximal in $R \equiv_{d f} x \in \operatorname{dom} R \&(\forall y)[\langle x, y\rangle \in R \Rightarrow\langle y, x\rangle$

$$
e \text { is cofinal in } R \equiv_{d f}(\forall x)\left[\phi_{e}(\bar{x}) \text { is defined] \& }(\forall x, y)\right.
$$

$\left[x \leqq y \Leftrightarrow\left\langle\phi_{e}(\bar{x}), \phi_{e}(\bar{y})\right\rangle \in R\right] \&(\forall z)\left[z \in \operatorname{dom} R \Rightarrow(\exists x)\left[\left\langle z, \phi_{e}(\bar{x})\right\rangle \in R\right]\right]$.

The $f^{*}$ operation is now defined.

Definition. Given any relation $R, f^{*}(R)$ is defined as follows:

(i) In case $(\exists x)[x$ maximal in $R]: f^{*}(R)=R \cup\left\{\left\langle x, 2^{y}\right\rangle \mid\langle x, y\rangle \in R\right\}$ $\cup\left\{\left\langle 2^{x}, 2^{y}\right\rangle \mid\langle x, y\rangle \in R\right\}$.

(ii) In case $(\forall x)[x$ not maximal in $R]$ and $(\exists e)[e$ cofinal in $R]: f^{*}(R)$ $=R \cup\left\{\left\langle x, 3 \cdot 5^{e}\right\rangle \mid\right.$ all $x$ in dom $R$, all $e$ cofinal in $\left.R\right\} \cup\left\{\left\langle 3 \cdot 5^{e}, 3 \cdot 5^{e^{\prime}}\right\rangle \mid\right.$ all $e$ and $e^{\prime}$ cofinal in $\left.R\right\}$.

(iii) Otherwise: $f^{*}(R)=R$.

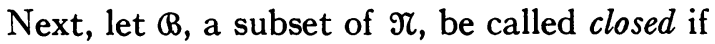

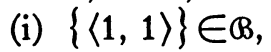

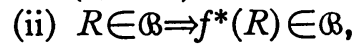

(iii) $Q \subseteq \complement \Rightarrow \cup_{R \in Q} R \in B$.

(5) This formulation of the definition of $<_{0}$ was obtained independently by Wang who ssed it to make a brief calculation of an upper bound for $O$ in the analytic hierarchy of $\left[\mathrm{K}_{2}\right]$, (presented at Cornell Institute for Mathematical Logic in Summer 1957). The above definition, (not Wang's application) was given by one of the authors in a seminar at M.I.T. in Spring 1957. 
Clearly $\Re$ is closed. The intersection of all such closed $B$ is (see $\$ 4$ ) a wellordered chain with maximal element. Let $R^{\prime}$ be this maximal element. Let $\widetilde{R}=\left\{\langle x, y\rangle \mid\langle x, y\rangle \in R^{\prime} \&\langle y, x\rangle \notin R^{\prime}\right\}$. Then we have

TheOREM 3. $\tilde{R}=<_{s}$.

Proof. See $\$ 4$. Technique is similar to proof of Theorem 2.

In both the definition of $S_{3}$ as $\hat{R}$ and $S_{1}$ as $\widetilde{R}$, one should note the parallel between, on the one hand, closure rules for the second number class and, on the other hand, cases (i)-(iv) of the definition of admissibility for $\hat{R}$ and cases (i)-(iii) of the definition of $f^{*}$ for $\tilde{R}$. The extensions to be discussed in $\$ \S 3-5$ will build on this analogy.

While the definitions of $\hat{R}$ and $\widetilde{R}$ are not necessary for a careful development of the properties of $S_{1}$ and $S_{3}$, their form is more useful for carrying out rigorous proof. Indeed, when extensions are attempted, work based on "inductive definitions" can lead to error. (See §3.)

3. A constructive third number class. We first look briefly at a known extension of $S_{3}$. In [AK] Addison and Kleene describe a "constructive third number class" of notations by straightforward analogy with the closure rules for the third number class of ordinals. They use an inductive definition as follows:

Let $<_{1}$ be the subsystem of $S_{3}$ giving notations to the finite ordinals. Let $O_{1}$ be the set of these notations.

Let " $<_{2}$ " be another name for $<_{0}$, and let " $\mathrm{O}_{2}$ " be another name for $O$. We simultaneously define $<_{2}^{2}$ (our new system), and $O_{2}{ }^{2}$, the set of notations (domain) of $<_{2}^{2}$.

(i) $1 \in O_{2}^{2}$,

(ii) $x \in O_{2^{2}} \Rightarrow\left[2^{x} \in O_{2^{2}} \& x<2_{2}^{2} 2^{x}\right]$,

(iii) $\left[x<_{2}^{2} y \& y<_{2}^{2} z\right] \Rightarrow x<_{2}^{2} z$,

(iv) $\left[(\forall x)\left[x \in O_{1} \Rightarrow \phi_{\theta}(x)\right.\right.$ defined $\left.] \&(\forall x, y)\left[x<_{1} y \Rightarrow \phi_{e}(x)<2^{2} \phi_{e}(y)\right]\right]$ $\Rightarrow 3 \cdot 5^{e} \in O_{2^{2}} \&(\forall x)\left[x \in O_{1} \Rightarrow \phi_{\theta}(x)<2_{2}^{2} 3 \cdot 5^{e}\right]$,

(v) $\left[(\forall x)\left[x \in O_{2} \Rightarrow \phi_{e}(x)\right.\right.$ defined $\left.] \&(\forall x, y)\left[x<_{2} y \Rightarrow \phi_{\theta}(x)<_{2}^{2} \phi_{e}(y)\right]\right]$ $\left.\Rightarrow 3^{2} 5^{\circ} \in O_{2}{ }^{2} \&(\forall x)\left[x \in O_{2} \Rightarrow \phi_{6}(x)<{ }_{2}^{2} 3^{2} 5^{e}\right]\right]$.

Note that use of only conditions (i)-(iii) would produce the system $<_{1}$, and that use of only conditions (i)-(iv) would produce the system $<_{2}$ (i.e. the system $S_{3}$ ). It is clear how additional conditions could be added to give constructive versions of all number classes of finite order.

Before undertaking any further extension, it is well to examine some of the properties of $<_{2}{ }^{2}$. Unfortunately, the desirable characteristics of $S_{3}$ (see \$2) are lost, and a number of irregularities appear that cast doubt on the usefuless of further extensions of this kind. We list some of these irregularities here. In what follows, $i$ is a fixed Gödel number for the identity function, and $|x|$ is the ordinal having $x$ as notation in $<_{2}^{2}$. 
(1) $<_{2}{ }^{2}$ does not have the tree-like structure of $<_{o}$, (i.e. the linear ordering of the predecessors of any $x$ in $<0$ ). For example, take $3^{2} 5^{i}$; this is a common upper bound in $<_{2}^{2}$ for any two incomparable notations in $<_{o}$, (the latter remaining incomparable in $<_{2}^{2}$ ).

(2) It is not true that every notation in $<_{2}^{2}$ but not in $<_{0}$ is comparable with all notations in $<_{o}$. There are notations in $<_{2}^{2}$ for the least nonconstructive ordinal (i.e. $\left|3^{2} 5^{i}\right|$ ) which are incomparable with certain notations in $<_{o}$. For example take $3^{2} 5^{j}$ where $\phi_{j}$ is the function $\lambda x\left[3 \cdot 5^{i}+o x\right]$ and to is the function defined in $\left[K_{1}\right]$. Then $\left|3^{2} 5^{i}\right|=\left|3^{2} 5^{i}\right|$ and for any $y$ in $<_{o}$, $y<2^{2} 3^{2} 5^{i} \Rightarrow y$ is comparable with $3 \cdot 5^{i}$ in $<_{o}$. Hence the infinitely many notations in $<_{o}$ which are incomparable with $3 \cdot 5^{i}$ in $<_{o}$ are incomparable with $3^{2} 5^{j}$ in $<_{2}^{2}$.

(3) Let $R$ be a linear subordering of $<_{2}^{2}$ between given endpoints $x$ and $y$. Let us say that $R$ is complete if $(\forall z)$ [ $z$ comparable with all members of $R \& z$ between $x$ and $y \Rightarrow z$ in $R$ ]. Then there exist two linear suborderings of $<_{2}{ }^{2}$ with common endpoints such that they are both complete, yet have distinct order types. In particular, take 1 and $3^{2} 5^{i}$ as end points. Since there are linear "branches" of $<_{o}$ that terminate at ordinal $\omega^{2}$ (see $\left.\left[K_{1}\right]\right)\left(^{6}\right)$, there are complete linear orderings between these endpoints of order type $\omega^{2}+1$. From Theorem 4 of $[\mathrm{S}]$, there are also linear orderings of the type of $\left|3^{2} 5^{i}\right|+1$.

(4) There exists a notation $3^{2} 5^{e}$ in $<_{2}^{2}$ such that the map $\phi_{\theta}$, defined on $O\left(=O_{2}\right)$ is not well defined with respect to ordinal numbers. In particular, there may be an $x$ and $y$ in $O$ such that $|x|=|y|$ but $\left|\phi_{e}(x)\right| \neq\left|\phi_{e}(y)\right|$. For example, take $\phi_{e}$ such that, for any $z, \phi_{\theta}(z)=2^{z}$ if $z \in\left\{3 \cdot 5^{i}, 2^{3 \cdot 5^{i}}, 2^{2^{3 \cdot 5 i}}, \cdots\right\}$, and $\phi_{e}(z)=z$ otherwise. Then the hypothesis of condition (v) is satisfied, and $3^{2} 5^{e}$ is in $<_{2^{2}}$. In fact, $\left|3^{2} 5^{e}\right|=\left|3^{2} 5^{i}\right|$. However, letting $j \neq i$ be another Gödel number for the identity function, we have $\left|3 \cdot 5^{j}\right|=\left|3 \cdot 5^{i}\right|$ but $\left|\phi_{e}\left(3 \cdot 5^{j}\right)\right|$ $\neq\left|\phi_{\theta}\left(3 \cdot 5^{i}\right)\right|$.

These examples suggest various counter-intuitive difficulties that may hinder further extensions of $S_{3}$. This is particularly evident when we consider that in any extension paralleling the generation of ordinal number classes out to the least inaccessible ordinal, every notation will itself be expected to appear later as the "index" of a "number class" (just as every $\alpha$ appears as index of some type number $\tau_{\alpha}$ in $\$ 1$ ). Thus the situation may become unusually complex. As we shall see, extension of $S_{1}$ proves to be simpler and more natural. (The superior features of $S_{3}$ over $S_{1}$ are lost anyway in extension of $S_{3}$.) This extension of $S_{1}$ will be given in $\$ 4$. The problem of making extensions of $S_{3}$ beyond that suggested by Kleene and Addison remains challenging as an independent exercise. Such an extension of $S_{3}$ is described in $\S 5$, and considered further in $\$ 7$.

(8) This fact is stated without proof in $\left[K_{1}\right]$. A trivial proof follows from countability considerations. 
Another difficulty besetting the definition of extensions either of $S_{1}$ or $S_{3}$ is the fact that with closure rules such as (iv) and (v) above, the larger a given "number class" is, the smaller will be the succeeding "number class". (E.g. in (v), the more members of $O_{2}$, the fewer $e$ to which rule (v) can apply.) This prevents a definition by intersection as simple as that for $S_{3}$ given in $\S 2$. Failure to note this has led to several erroneous attempts to extend $S_{3}$.

It is instructive to look at an example of such plausible but erroneous definitions. Consider the intersection of all ternary relations $P$ such that $P \subseteq N \times N \times N$ and

(i) $\langle 1,1,2\rangle \in P$,

(ii) $\langle x, 1, y\rangle \in P \Rightarrow\left\langle x, y, 2^{y}\right\rangle \in P$,

(iii) $\langle x, 1, y\rangle \in P \Rightarrow\langle y, 1,2\rangle \in P$,

(iv) $\langle w, x, y\rangle \in P \&\langle w, y, z\rangle \in P \Rightarrow\langle w, x, z\rangle \in P$,

(v) $\left[(\exists x)[\langle x, y, z\rangle \in P] \&(\forall u, v)\left[\langle y, u, v\rangle \in P \Rightarrow \phi_{e}(u)\right.\right.$ and $\phi_{e}(v)$ defined $\left.\left.\&\left\langle z, \phi_{e}(u), \phi_{e}(v)\right\rangle \in P\right]\right] \Rightarrow(\forall u, v)\left[\langle y, u, v\rangle \in P \Rightarrow\left\langle z, \phi_{e}(u), 3^{y} 5^{e}\right\rangle \in P\right]$. Call this intersection $\tilde{P}$. Our hope would be that $\{\langle u, v\rangle \mid\langle x, u, v\rangle \in \widetilde{P}\}$ could be taken as a system of notations for the "constructive number class" of "index $x$ ", and in particular, that $\{\langle u, v\rangle \mid\langle 2, u, v\rangle \in \widetilde{P}\}$ would be $<_{o}$. Unfortunately, however, $\tilde{P}$ itself is not closed under (i) $-(\mathrm{v})$, and fails even to provide the usual notations of $<_{o}$. Let $\tilde{e}$ be a fixed Gödel number such that $\phi_{\tilde{e}}(x)=x$ if $x \in O_{1}$, and $\phi_{\tilde{e}}(x)$ is undefined otherwise. Then $\left\langle 1,3 \cdot 5^{\tilde{e}}\right\rangle$ is in $\left\langle_{o}\right.$. Furthermore, as is easily seen, the hypothesis of condition (v) is satisfied with $e=\tilde{e}, y=1, z=2$, and $P=\widetilde{P}$. However, the conclusion of (v) is not satisfied, $3 \cdot 5^{\tilde{e}}$ is not a notation in $\widetilde{P}$, and therefore $\widetilde{P}$ does not give an extension $<_{o}$ as desired. For define $P^{\prime}=\left\{\langle u, v, w\rangle \mid u \neq 3 \cdot 5^{\tilde{e}} \& v \neq 3 \cdot 5^{\tilde{e}} \& w \neq 3 \cdot 5^{\tilde{e}}\right\}$. If we can show $P^{\prime}$ satisfies (i) $-(v)$, then we will have $\tilde{P} \subseteq P^{\prime}$ and that $3 \cdot 5^{\tilde{c}}$ does not occur in $\tilde{P}$. Clearly $P^{\prime}$ satisfies (i)-(iv). Assume the conclusion of (v) false for $P^{\prime}$ and some $y, z$, and $e$. Then there must be $u$ and $v$ such that $\langle y, u, v\rangle \in P^{\prime}$ but $\left\langle z, \phi_{e}(u), 3^{y} 5^{e}\right\rangle \notin P^{\prime}$. If $\left\langle z, \phi_{e}(u), 3^{y} 5^{e}\right\rangle \notin P^{\prime}$, then either $z$ or $\phi_{e}(u)$ or $3^{y} 5^{e}$ must equal $3 \cdot 5^{\tilde{c}}$. If $z=3 \cdot 5^{\tilde{\varepsilon}}$, then the first part of the antecedent of $(v)$ is false. If $\phi_{e}(u)=3 \cdot 5^{\tilde{\varepsilon}}$, then the second part of the antecedent is false. If $3^{y} 5^{e}=3 \cdot 5^{\tilde{\varepsilon}}$, then $y=1$ and $e=\tilde{e}$. In this last case, the second part of the antecedent is still false since, for example, $\left\langle 1,7,2^{7}\right\rangle \in P^{\prime}$, but $\phi_{\tilde{e}}(7)$ is undefined. Thus in every case the antecedent of $(v)$ is false. Hence $P^{\prime}$ satisfies condition (v).

4. Extension of $S_{1}$. We now define an extension of $S_{1}$ that generalizes the explicit definition of $S_{1}$ given at the end of $\$ 2$. Our definitions and proofs are arranged so that mention of ordinals is avoided until final application of Theorem 1 to the constructed system. In $\$ 6$ of [CK], pp. 20-21, Church and Kleene suggest what is, in effect, an extension of $S_{1}$. (Formulas of a lambda calculus, rather than integers, are used as notations.) Our extended system can be viewed as a precise reformulation of the Church-Kleene suggestion, suitable for the computations to be made in $\$ \$ 6$ and 7 below. We begin with a set-theoretic lemma. $\Re$, as before, is the set of all subsets of $N \times N$. 
Let $f^{*}$ be a map of $\mathfrak{N}$ into $\mathfrak{N}$ with the property that $R \subseteq f^{*}(R)$ for all $R \in \Re$. Let a fixed $R_{0} \in \Re$ be given. Consider the intersection of all collections $\mathfrak{N} \subseteq \mathfrak{N}$ satisfying the conditions:

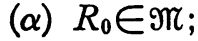

(B) $R \in \Re \Rightarrow f^{*}(R) \in \mathscr{M}$;

$(\gamma) Q \subseteq \Re \& Q \neq \varnothing \Rightarrow U\{R \mid R \in Q\} \in \Re$.

We call this intersection $D_{R_{0}}$, or more briefly, $D$.

By a chain, we mean a collection of sets linearly ordered under $\subseteq$. The set $U\{T \mid T \in D \& T \subseteq R \& T \neq R\}$ we call $P(R)$. Note that $(\alpha),(\beta)$ and $(\gamma)$ are satisfied by $D(=\Re)$. It directly follows that $R_{0} \subseteq R$ for all $R \in D$, and hence, by $(\gamma)$, that $P(R) \in \mathbb{D}$ for every $R \in D$ such that $R \neq R_{0}$.

LEMMA 1. (a) $D$ is a well-ordered chain;

(b) if $R \in D$, then either $R=R_{0}, R=P(R)$, or $R=f^{*}(P(R))$;

(c) D has a maximal element $\bar{R}$ such that $f^{*}(\bar{R})=\bar{R}$.

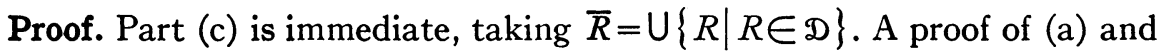
(b) using transfinite induction over ordinals would be a standard set theoretical exercise. We present, in outline, a proof that avoids ordinals, since it is closely related to certain subsequent proofs.

Let a set $R \in D$ be called admissible if:

(i) $(\forall T)[T \in D \Rightarrow T \subseteq R$ or $R \subseteq T]$;

(ii) $\{T \mid T \in \mathscr{D} \& T \subseteq R\}$ is a well-ordered chain;

(iii) $(\forall T)\left[T \in D \& T \subseteq R \Rightarrow T=R_{0}\right.$ or $T=P(T)$ or $\left.T=f^{*}(P(T))\right]$.

Let $a$ be the collection of admissible sets. By definition $a \subseteq D$. If we show that $Q$ satisfies $(\alpha),(\beta)$ and $(\gamma)$, then we will have $D \subseteq Q$, and hence $Q=D$. Parts (a) and (b) of the lemma will then follow from conditions (i), (ii) and (iii) on members of $a$.

$R_{0} \in Q$, since $R_{0}$ is contained in every member of $D$. Hence $Q$ satisfies $(\alpha)$.

Let $R$ be admissible; we wish to show $R^{\prime}=_{d f} f^{*}(R)$ is admissible. Let $S_{R}=_{d f}\{T \mid T \in D \& T \subseteq R\} \cup D_{R^{\prime}}$. (Here $D_{R^{\prime}}$, is defined from $R^{\prime}$ in the same way that $D\left(=D_{R_{0}}\right)$ is defined from $R_{0}$.) We first show that $\delta_{R}$ satisfies $(\alpha),(\beta)$ and $(\gamma) . \delta_{R}$ satisfies $(\alpha)$ immediately. By (ii) and (iii) $\delta_{R}$ satisfies $(\beta)$. (Consider separate cases for $D_{R^{\prime}}$ and for $S_{R}-D_{R^{\prime}}$ ) Let $Q^{\prime} \subseteq S_{R}$ and let $Q=U\left\{T \mid T \in Q^{\prime}\right\}$. Then, considering separately the cases $Q \subseteq R$ and $R^{\prime} \subseteq Q$, we have from (ii) and (iii) that $Q \in \delta_{R}$. Hence $\delta_{R}$ satisfies $(\gamma)$. We therefore now have $D \subseteq \delta_{R}$. From this and the definition of $\delta_{R}$, it follows that $R^{\prime}$ satisfies (i), (ii) and (iii) and is therefore admissible. Hence $Q$ satisfies $(\beta)$.

If $Q \subseteq Q$, then, setting $R^{\prime \prime}=U\{T \mid T \in \mathcal{Q}\}$, we can show by exactly similar argument to the preceding case that $R^{\prime \prime}$ is admissible. Hence $Q$ satisfies $(\gamma)$. Q.E.D.

Corollary. $R, T \in D \& R \subseteq T \& R \neq T \Rightarrow f^{*}(R) \subseteq T$.

Proof. By construction of $S_{R}=D$. 
Our extension of $S_{1}$ will be called $C$. The motivational comments made right after Theorem 2 in $\$ 2$ are also appropriate here. We make preliminary definitions for any $R$ and $T$ in $\Re$.

Definition. (i) $u \in R \equiv_{d f} u \in d o m R$.

(ii) $u$ is maximal in $R \equiv_{d f} u \in R \&(\forall z)[\langle u, z\rangle \in R \Rightarrow\langle z, u\rangle \in R]$.

(iii) $a$ is an index in $R \equiv_{d f}(\exists z)\left[3^{a} 5^{z} \in R\right]$.

(iv) $r e l^{R} a=_{d f}\left\{\langle u, v\rangle \mid\langle u, v\rangle \in R \&(\forall x)(\forall z)\left[\langle x, v\rangle \in R \Rightarrow x \neq 3^{a} 5^{z}\right]\right\}$.

[Motivation. (iii) means (by analogy and for appropriate $R$ ) that notations appear in $R$ whose "limit type" is the "type number" whose index is $a$. (iv) is the restriction of $R$ to predecessors of this "type number of index $a$ ".]

(v) e maps Rorder-isomorphic into $T$ ( “OP $(e, R, T)$ ") $\equiv_{d j}(\forall u)\left[u \in R \Rightarrow \phi_{\theta}(u)\right.$ defined $] \&(\forall u, v)\left[\langle u, v\rangle \in R \Leftrightarrow\left\langle\phi_{e}(u), \phi_{e}(v)\right\rangle \in T\right]$.

(Note the use of $\Leftrightarrow$ instead of $\Rightarrow$ in (v).)

(vi) e maps $R$ cofinally into $T$ (“OPC $(e, R, T)$ ") $\equiv_{d f} O P(e, R, T) \&(\forall z)$ $\left[z \in T \Rightarrow(\exists u)\left[u \in R \&\left\langle z, \phi_{e}(u)\right\rangle \in T\right]\right]$.

Notation. $C_{1}(y, R) \equiv_{d f} y$ maximal in $R$.

$C_{2}(a, e, R) \equiv_{d f} a$ is an index in $R \& O P C\left(e, r^{R} l^{R} a, R\right)$.

$C_{3}(b, e, R) \equiv_{d f} b \in R \& b$ not an index in $R \&(\forall x)[\langle x, b\rangle \in R \Rightarrow[\langle b, x\rangle \in R$ or $x$ an index in $R]]$ \& $O P(e, R, R)\left({ }^{7}\right)$.

Definition. Given any $R \in \mathfrak{N}, f^{*}(R)$ is defined as follows:

$\left(1^{*}\right)$ In case $(\exists y) C_{1}(y, R): f^{*}(R)=R \cup\left\{\left\langle x, 2^{y}\right\rangle \mid x \in R \& C_{1}(y, R)\right\}$ $\cup\left\{\left\langle 2^{y}, 2^{z}\right\rangle \mid C_{1}(y, R) \& C_{1}(z, R)\right\}$.

$\left(2^{*}\right)$ In case not- $\left.\exists y\right) C_{1}(y, \quad R) \&(\exists a, \quad e) C_{2}(a, \quad e, \quad R): f^{*}(R)$ $=R \cup\left\{\left\langle x, 3^{a} 5^{e}\right\rangle \mid x \in R \& C_{2}(a, e, R)\right\} \cup\left\{\left\langle 3^{a} 5^{e}, 3^{a^{\prime}} 5^{a^{\prime}}\right\rangle \mid C_{2}(a, e, R)\right.$ $\left.\& C_{2}\left(a^{\prime}, e^{\prime}, R\right)\right\}$.

$\left(3^{*}\right)$ In case not- $(\exists y) C_{1}(y, R)$ \& not- $(\exists a, e) C_{2}(a, e, R) \&(\exists b)[b \in R$ $\& b$ not an index in $R]: f^{*}(R)=R \cup\left\{\left\langle x, 3^{b} 5^{e}\right\rangle \mid x \in R \& C_{3}(b, e, R)\right\}$ $\cup\left\{\left\langle 3^{b} 5^{e}, 3^{b^{\prime}} 5^{e^{\prime}}\right\rangle \mid C_{3}(b, e, R) \& C_{3}\left(b^{\prime}, e^{\prime}, R\right)\right\}$.

$\left(4^{*}\right)$ Otherwise : $f^{*}(R)=R$.

REMARK. Let $i$ be any Gödel number for the identity function; then $O P(i, R, R)$ is always true. Although $R \subseteq f^{*}(R)$, it is easy to show that $R \subseteq T$ does not in general imply $f^{*}(R) \subseteq f^{*}(T)$ for arbitrary relations $R$ and $T$.

Definition. $R_{0}={ }_{d f}\{\langle 1,1\rangle\} . D={ }_{d f} D_{R_{0}}$ as defined for Lemma 1 , using the $f^{*}$ defined immediately above. $P(R)$ is as defined before. Of course, Lemma 1 now applies.

[Motivation. The conditions $\left(1^{*}\right)$ and $\left(2^{*}\right)$ correspond to closure rule (ii) (before Result 10 in \$1) for generating ordinals (with the cases of successor and limit ordinal treated separately). Condition $\left(3^{*}\right)$ corresponds to the closure rule (iii) in §1.]

Lemma 2. For all $R \in D$ :

(7) $O P C(e, R, R)$ may seem more natural here than $O P(e, R, R)$, but the construction goes through satisfactorily, and equivalently, with the latter. 
(a) $u, v \in R \Rightarrow[\langle u, v\rangle \in R$ or $\langle v, u\rangle \in R]$;

(b) $u \in R \Rightarrow\langle u, u\rangle \in R$;

(c) $u$ maximal in $R \Leftrightarrow(\forall v)[v \in R \Rightarrow\langle v, u\rangle \in R]$;

(d) $\langle v, u\rangle \in(R-P(R)) \Rightarrow u$ maximal in $R$;

(e) $2^{u} \in R \Rightarrow u \in P(R)$;

(f) $u$ is maximal in the least $R$ containing $u$;

(g) $u \in R \Rightarrow u=1$ or $(\exists z)\left[u=2^{z}\right]$ or $(\exists a, e)\left[u=3^{a} 5^{e}\right]$.

Proof. (a) If false, let $R_{1}$ be the least member $R$ of $D$ for which, for some $u, v, u \in R_{1}, v \in R_{1},\langle u, v\rangle \notin R_{1}$ and $\langle v, u\rangle \notin R_{1}$. Then $R_{1} \neq R_{0}$. If $u, v$ both $\in P\left(R_{1}\right)$, then by the linear ordering of $D$, there is an $R_{2} \subseteq R_{1}, R_{2} \neq R_{1}$ such that $u, v \in R_{2}$, and hence $\langle u, v\rangle \notin R_{2}$ and $\langle v, u\rangle \notin R_{2}$ (since $R_{2} \subseteq R_{1}$ ), contrary to the choice of $R_{1}$. If, say, $u \notin P\left(R_{1}\right)$, then $R_{1}=f^{*}\left(P\left(R_{1}\right)\right)$ and $u$ must be introduced by $\left(1^{*}\right),\left(2^{*}\right)$ or $\left(3^{*}\right)$, which implies that $\langle w, u\rangle \in R_{1}$ for all $w \in R_{1}$; in particular $\langle v, u\rangle \in R_{1}$ contrary to the choice of $R_{1}$.

(b) This is immediate from (a).

(c) Assume $u$ maximal in $R$; then if $w \in R$ and $\langle w, u\rangle \notin R$, we must have $\langle u, w\rangle \in R$ by (a). But then by definition of maximality we have $\langle w, u\rangle \in R$. Hence $(\forall w)[w \in R \Rightarrow\langle w, u\rangle \in R]$. The converse part of (c) is immediate by definition of maximality.

(d) If $\langle v, u\rangle \in(R-P(R))$, then $R=f^{*}(P(R))$ and $\langle v, u\rangle$ is introduced by $\left(1^{*}\right),\left(2^{*}\right)$ or $\left(3^{*}\right)$. But each of these cases gives $(\forall w)[w \in R \Rightarrow\langle w, u\rangle \in R]$, hence by (c) $u$ is maximal.

(e) Take $R_{2}=$ the least $R$ with $2^{u} \in R$. Then, by Lemma $1, R_{2}=f^{*}\left(P\left(R_{2}\right)\right)$. Hence $2^{u}$ must appear in $R_{2}$ via $\left(1^{*}\right)$. But this implies $u \in P\left(R_{2}\right)$. Now $2^{u} \in R$ $\Rightarrow R_{2} \subseteq R \Rightarrow P\left(R_{2}\right) \subseteq P(R) \Rightarrow u \in P(R)$.

(f) $u$ must appear in $R$ via $\left(1^{*}\right),\left(2^{*}\right)$ or $\left(3^{*}\right)$. Hence by (d), $u$ is maximal in $R$.

(g) If false for some $R$, take the least such $R$. A contradiction is immediate from $\left(1^{*}\right),\left(2^{*}\right)$ and $\left(3^{*}\right)$. Q.E.D.

Definition. $R, \quad T$ are coherent $\equiv_{d f}(\forall u)[u \in R \& u \in T \Rightarrow(\forall v)[\langle v, u\rangle$ $\in R \Leftrightarrow\langle v, u\rangle \in T]]$.

Lemma 3. For any $R, T \in D, R, T$ are coherent.

Proof. Assume false; let $T_{1}$ be the least $T \in D$ for which there is an $R \in D$ such that $R \subseteq T$, and $R, T$ not coherent. Let $R_{1}$ be the least such $R$. We obtain a contradiction.

Sublemma 1. If $a$ is an index in $R_{1}$, then $\operatorname{rel}^{R_{1}} a=\operatorname{rel}^{P\left(T_{1}\right)} a$.

Proof. $R_{1}$ and $P\left(T_{1}\right)$ are coherent by assumption, and $R_{1} \subseteq P\left(T_{1}\right)$. Assume $\langle u, v\rangle \in r e l^{R_{1}} a$. Then $\langle u, v\rangle \in R_{1}$ and for no $e$ does $\left\langle 3^{a} 5^{e}, v\right\rangle \in R_{1}$. It follows that $\langle u, v\rangle \in P\left(T_{1}\right)$ and, by coherence, that for no $e$ does $\left\langle 3^{a} 5^{e}, v\right\rangle \in P\left(T_{1}\right)$. Hence $r e l^{R_{1}} a \subseteq r e l^{P\left(T_{1}\right)} a$. Assume $\langle u, v\rangle \notin r e l^{R_{1}} a$. Case 1: $\langle u, v\rangle \in R_{1}$. Then $\left\langle 3^{a} 5^{e}, v\right\rangle \in R_{1}$ 
for some $e$. But then $\left\langle 3^{a} 5^{e}, v\right\rangle \in P\left(T_{1}\right)$. Hence $\langle u, v\rangle \operatorname{Frel}^{P\left(T_{1}\right)} a$. Case 2: $\langle u, v\rangle$ $\notin R_{1}$. But $3^{a} 5^{e} \in R_{1}$ for some $e$. Hence if $\langle u, v\rangle \in P\left(T_{1}\right),\left\langle 3^{a} 5^{e}, v\right\rangle \in P\left(T_{1}\right)$, by Lemmas $2 \mathrm{c}$ and $2 \mathrm{~d}$. Hence $\langle u, v\rangle \notin r e l^{P\left(T_{1}\right)} a$. Thus, in either case, $\operatorname{rel}^{P\left(T_{1}\right)} a$ $\subseteq$ rel $^{R_{1}} a$.

Sublemma 2. $u$ maximal in $R_{1} \Rightarrow u$ not maximal in $T_{1}$.

Proof. Assume $u$ maximal in both $R_{1}$ and $T_{1}$. Then by $\left(1^{*}\right), 2^{u} \in f^{*}\left(R_{1}\right)$ $\subseteq T_{1}$. Hence by Lemma $2 \mathrm{c},\left\langle 2^{u}, u\right\rangle \in T_{1}$. Now by Lemma $2 \mathrm{e}, T_{1}$ is not coherent with the least $R$ containing $u$. Hence $R_{1}$ is the least $R$ containing $u$, and $\left\langle 2^{u}, u\right\rangle \notin R_{1}$. By assumption of coherence, $\left\langle 2^{u}, u\right\rangle \notin P\left(T_{1}\right)$. Hence $\left\langle 2^{u}, u\right\rangle$ appears in $T_{1}$ by $\left(1^{*}\right),\left(2^{*}\right)$ or $\left(3^{*}\right)$. Case $1: u=2^{v}$. Then $v$ maximal in $P\left(R_{1}\right)$ and in $P\left(T_{1}\right)$. Hence, by similar argument to above, $\left\langle 2^{v}, v\right\rangle \in P\left(T_{1}\right)$. But then $P\left(T_{1}\right)$ is not coherent with the least $R$ containing $v$, contrary to the choice of $T_{1}$. Case 2: $u=3^{a} 5^{e}$. Then $\left\langle 2^{u}, u\right\rangle$ appears in $T_{1}$ via $\left(2^{*}\right)$, since $a$ is an index in $P\left(T_{1}\right)$. Hence $O P C\left(e, \operatorname{rel}^{P\left(T_{1}\right)} a, P\left(T_{1}\right)\right)$. But by Sublemma $1, \operatorname{rel}^{P\left(T_{1}\right)} a$ $=\operatorname{rel}^{R_{1}} a$ (since $a$ is also an index in $R_{1}$ ). Therefore $(\exists v)\left[v \in r e l^{R_{1}} a \&\left\langle 3^{a} 5^{e}, \phi_{e}(v)\right\rangle\right.$ $\left.\in T_{1}\right]$, by definition of $O P C$. But $\phi_{e}(v) \in P\left(R_{1}\right)$, since $u$ appears in $R_{1}$ via $\left(2^{*}\right)$ or $\left(3^{*}\right)$. However $3^{a} 5^{e} \notin P\left(R_{1}\right)$ and hence $\left\langle 3^{a} 5^{e}, \phi_{e}(v)\right\rangle \notin P\left(R_{1}\right)$. Thus coherence of $P\left(R_{1}\right)$ and $P\left(T_{1}\right)$ is violated; and the proof of the sublemma is complete.

To complete the proof of the lemma, take $u \in R_{1},\langle v, u\rangle \in T_{1},\langle v, u\rangle \notin R_{1}$, by assumption that $R_{1}, T_{1}$ not coherent. Then $T_{1}$ must be the least relation in $D$ with $\langle v, u\rangle$ (by choice of $T_{1}$ ), and $R_{1}$ must be the least relation with $u$. But this means, by Lemmas $2 \mathrm{~d}$ and $2 \mathrm{f}$, that $u$ is maximal in both $R_{1}$ and $T_{1}$ in violation of Sublemma 2. Q.E.D.

CoROllaRy. For $R$ and $T$ in $D$ :

(i) $u$ maximal in $R \Leftrightarrow u \in R \& u \in P(R)$;

(ii) $a$ index in both $R$ and $T \Rightarrow r e l^{R} a=r e l^{T} a$.

LEMMA 4. $R \in D \Rightarrow R$ is transitive.

Proof. Assume not. Take $R_{1}$ to be the least nontransitive member of $D$. Then for some $u, v, w:\langle u, v\rangle \in R_{1},\langle v, w\rangle \in R_{1}$, and $\langle u, w\rangle \notin R_{1}$. By Lemma $2 \mathrm{c}$, $w$ not maximal in $R_{1}$. By Lemma $2 \mathrm{~d},\langle v, w\rangle \in P\left(R_{1}\right)$. By corollary to Lemma 3, $v$ not maximal in $R_{1}$. Hence, by Lemma $2 \mathrm{~d},\langle u, v\rangle \in P\left(R_{1}\right)$. Hence $P\left(R_{1}\right)$ is not transitive, contrary to choice of $R_{1}$. Q.E.D.

Definition. $R$ has the descending chain condition $(D C C) \equiv_{d f}$ there is no infinite sequence $x_{0}, x_{1}, \cdots$ such that for all $i,\left\langle x_{i+1}, x_{i}\right\rangle \in R \&\left\langle x_{i}, x_{i+1}\right\rangle \notin R$.

LEMma 5. $R \in \mathbb{D} \Rightarrow R$ has the $D C C$.

Proof. If false, take $R_{1}=$ the least $R$ with a descending chain. At most the first member of the chain can be maximal in $R_{1}$. By coherence, $P\left(R_{1}\right)$ must have a descending chain,-a contradiction. Q.E.D.

We now define our extension of $S_{1}$.

Definition. $\lesssim_{c}=_{d f} \cup\{R \mid R \in D\}=\bar{R} . x<_{c} y \equiv_{d f} x \lesssim_{c} y \&$ not $y \lesssim_{c} x$. 
THEOREM 4. $<_{c}$ is a system of notations.

Proof. By Lemmas 2, 3, 4 and $5,<_{C}$ is a well-ordered partial ordering of integers. Q.E.D.

Let " ||$c$ " denote the mapping from $<_{c}$ given by Theorem 1 . The structure of $<_{c}$ is elucidated in the following theorem, where we see that $<_{c}$ gives a linear well-ordering of certain equivalence classes of incomparable elements, with the elements of each class mapped onto the same distinct ordinal by ||$c$.

Definition. $\left.x \approx_{c} y \equiv_{d f} x \lesssim_{c} y \& y\right\rfloor_{c} x . C=_{d f} \operatorname{dom}\left(<_{c}\right)$.

Theorem 5. For $x, y \in C$ :

(i) $x<_{c} y$ or $x \approx_{c} y$ or $y<_{c} x$.

(ii) $x<c y \Leftrightarrow|x| c<|y| c$.

(iii) $x \approx_{c} y \Leftrightarrow|x|_{c}=|y| c$.

Proof. (i) follows from Lemmas 1 and $2 \mathrm{a}$. By Lemma $4, x \approx_{c} y \Rightarrow\left[\left\{z \mid z<_{c} x\right\}\right.$ $\left.=\left\{z \mid z<_{c} y\right\}\right]$. Hence, by the construction of Theorem $1, x \approx_{c} y \Rightarrow|x| c$ $=|y|_{c}$, and $x<_{c} y \Rightarrow|x|_{c}<|y|_{c}$. (ii) and (iii) now follow. Q.E.D.

The structural analogy between $<_{c}$ and the accessible ordinals is indicated in the sequence of results about $<_{c}$ given below as Lemma 6 . As will be seen, the ordering $<_{a}$ (to be defined) plays the role of the " $|a| c$ th number class" (or, in case $|a|_{c}<\omega$, the " $|a|_{c}+1$ st number class"), and $\left|3^{a} 5^{i}\right|_{c}$ plays the role of the " $|a| c$ th infinite type number" (where $i$ is any fixed Gödel number for the identity function).

Definition. For $a \in C: \lesssim_{a}={ }_{d f} P(R)$ where $R$ is the least member of $D$ containing $a$ as index. ( $\left(3^{a} 5^{i}\right.$ will be in $R$.)

$$
x<_{a} y \equiv_{d f} x \lesssim_{a} y \& \text { not } y \lesssim_{a} x .
$$

LEMMA 6. (a) $\lesssim_{a}=\operatorname{rel} \lesssim c a$.

(b) $a \in C \Rightarrow a$ is an index in $\lesssim c$.

(c) $a \in C \& O P\left(e, \lesssim_{a}, \lesssim_{c}\right) \Rightarrow 3^{a} 5^{e} \in C$.

(d) $a \in C \Rightarrow$ not-OPC $\left(e, \lesssim_{a}, \lesssim_{c}\right)$.

(e) $a<_{c} b \& O P\left(e, \lesssim_{a}, \lesssim_{b}\right) \Rightarrow 3^{a} 5^{e} \in \lesssim_{b} \& \operatorname{not}-O P C\left(e, \lesssim_{a}, \lesssim_{b}\right)$.

(f) $<_{a_{1}}=<_{a_{2}} \Leftrightarrow\left|a_{1}\right|_{c}=\left|a_{2}\right| c$.

Proof. (a) follows by definition and Lemma 3. (b), (c), (d) and (e) follow from $\left(2^{*}\right),\left(3^{*}\right)$ and the fact that $f^{*}\left(\lesssim_{c}\right)=\lesssim_{c}$. (f) follows from $\left(3^{*}\right)$ and (iii) of Theorem 5. Q.E.D.

From the preceding results, we see how the system of notations $C$ is analogous, in its effective structure, to the number classes of accessible ordinals as generated by closure rules (i)-(iii) of $\$ 1$. Hence the segment of the second number class covered by $C$ (see Theorem 1) can itself be viewed as a constructive version, in this sense, of the segment of all accessible ordinals. By Lemma $6 f$, the segments of countable ordinals constituting the 
successive "constructive number classes" may be consistently indexed by ordinals rather than by ordinal notations.

Our main further results about $C$ occur in $\$ \$ 6$ and 7 below. Certain alternative approaches to the extension of $S_{1}$, certain problems concerning them, and certain further extensions of $C$ will be considered in $\$ 9$.

5. Extension of $S_{3}$. We next define an extension of $S_{3}$ that includes and goes beyond the " 3 rd constructive number class" of Kleene and Addison described in $\S 3$ above. Like the extension $C$ of $S_{1}$, it will be analogous in structure to the segment of accessible ordinal numbers. As in $\$ 4$, our definition will have a conventional set theoretical form that avoids "informal" transfinite induction; and, as in $\$ 4$, the material is arranged so that ordinal numbers are not used in the definition.

In outline, the extension will go as follows.

(i) If $x$ is already obtained, include $2^{x}$ as a successor of $x$.

(ii) If $I_{x}$ is the set of notations constituting the "number class of index $x$ " and if $\phi_{e}$ maps $I_{x}$ order-preserving into the notations already obtained, then $3^{x} 5^{e}$ must be included as an immediate successor to the images $\phi_{e}\left(I_{x}\right)$.

(iii) If the notations already obtained are closed under (i) and (ii), then call them $I_{y}$ where $y$ is an immediate successor to notations previously used as indices. ((ii) can always be applied immediately after (iii) by introducing $3^{y} 5^{i}$ where $i$ is a Gödel number for the identity function.)

Several difficulties arise in making this definition precise. Some have been indicated in the discussion of $\$ 3$. We mention two more here.

(a) The definition must allow sufficiently complex "dovetailings" of (i), (ii) and (iii). Thus the system must be arranged to include $3 \cdot 5^{\bar{c}}$ where $\phi_{\bar{e}}(1)$ $=3 \cdot 5^{i}, \cdots, \phi_{\bar{\varepsilon}}\left(2^{n}\right)=3^{\phi_{\bar{c}}^{(n)}} 5^{i}, \cdots$, even though $3 \cdot 5^{\bar{z}}$ does not appear until the "number class" of index $3 \cdot 5^{\bar{c}}$. (See parenthetical comment following Result 9 of $\$ 1\left({ }^{8}\right)$.)

(b) In order to prove the desired properties of our extension (e.g. that it is a system of notations), we have found it necessary to incorporate into the basic definition a certain regularity condition, namely that any notation of the form $3^{x} 5^{e}$ must be comparable with (and follow) the notation $x$. Whether or not the proofs can be carried through if this condition is dropped is an open question. Whether or not this condition can itself be proved as a theorem if it is dropped from the definition is an open question $\left({ }^{9}\right)$.

If the definition now to be given appears less simple than the outline above, it is because of difficulties (a) and (b). We give the definition, then list appropriate lemmas and theorems with brief indications of proof. Details

(8) Almost all proposed extensions which the present authors have seen have failed in this respect.

(॰) The regularity condition mentioned here is introduced by way of the condition " $(\exists m)\left[m \in \ln ^{A} y \&\left\langle y, \phi_{z}(m)\right\rangle \in R\right]^{n}$ in the definition of $\mathcal{C}(A, R)$ below, and by way of the condition " $\left\langle x\right.$, ind $\left.A_{\iota_{0}}\right\rangle \in \bigcup_{\imath \in J} \operatorname{rel} A$, for some ${ }_{0} \in J$ " in the definition of $R$ below. The regularity condition also appears necessary for the proof of Theorem 11 (and hence for Theorem 12) in $\$ 8$. 
are omitted, since our primary emphasis is on the system of $\S 4$. Proofs are given in full detail in $[\mathrm{Kr}]$.

$A, B, D, \cdots$ shall denote nonempty ternary relations (on the nonnegative integers) with the special property that for any relation $A,\left\langle x_{1}, y_{1}, z_{1}\right\rangle$ $\in A$ and $\left\langle x_{2}, y_{2}, z_{2}\right\rangle \in A \Rightarrow x_{1}=x_{2}$.

Definition. ind $A={ }_{d f} x$, where $(\exists y)(\exists z)[\langle x, y, z\rangle \in A]$.

$\operatorname{rel} A={ }_{d f}\{\langle y, z\rangle \mid\langle$ ind $A, y, z\rangle \in A\}$.

$\operatorname{rln}^{A} w={ }_{d f}\{\langle y, z\rangle \mid\langle y, z\rangle \in \operatorname{rel} A \&(\forall u)[u=z$ or $\langle u, z\rangle \in \operatorname{rel} A \Rightarrow(\forall a)(\forall b)$ $\left.\left[u=3^{a} 5^{b} \Rightarrow\langle a, w\rangle \in \operatorname{rel} A\right]\right]$.

As before, if $S$ is a binary relation, $x \in S$ abbreviates $(\exists y)[\langle x, y\rangle \in S$ or $\langle y, x\rangle \in S]$.

Definition. If $R$ is a binary relation, then

$$
\begin{aligned}
\mathcal{C}(A, R) \equiv & { }_{d r}\langle 1,2\rangle \in R \\
& \&\left[\langle 1, x\rangle \in R \Rightarrow\left\langle x, 2^{x}\right\rangle \in R\right] \\
& \&[\langle x, y\rangle \in R \&\langle y, z\rangle \in R \Rightarrow\langle x, z\rangle \in R] \\
& \&[[y=i n d A \text { or }\langle y, \text { ind } A\rangle \in \operatorname{rel} A] \\
& \&(\forall m)\left[m \in r n^{A} y \Rightarrow \phi_{z}(m) \text { defined }\right] \\
& \&(\forall m)(\forall n)\left[\langle m, n\rangle \in r \ln ^{A} y \Rightarrow\left\langle\phi_{z}(m), \phi_{z}(n)\right\rangle \in R\right] \\
& \&(\exists m)\left[m \in r \ln ^{A} y \&\left\langle y, \phi_{z}(m)\right\rangle \in R\right] \\
& \left.\Rightarrow(\forall m)\left[m \in r \operatorname{rn}^{A} y \Rightarrow\left\langle\phi_{z}(m), 3^{y} 5^{z}\right\rangle \in R\right]\right] .
\end{aligned}
$$

In the ternary relations $A$ that will ultimately appear, $\operatorname{rel} A$ will be the system of notations constituting the "number class" of index ind $A . \mathfrak{e}(A, R)$ asserts that $R$ is closed with respect to increasing sequences mapped from $A$ itself or from number classes contained in $A$.

Let $a$ represent a collection of ternary relations with the special property.

Definition. $R={ }_{d f}$ the intersection of all $a$ such that $\$ 3)$;

(1) there exists $A \in Q$ such that ind $A=1$ and $\operatorname{rel} A=<_{1}$ (see beginning

(2) if $A \in \mathrm{Q}$, then $A^{\prime} \in \mathrm{Q}$, where ind $A^{\prime}$ is $2^{\text {ind } A}$, and rel $A^{\prime}$ is the intersection of all $R$ such that $\mathfrak{e}(A, R)$;

(3) if $\left\{A_{\imath} \mid \imath \in J\right\} \subseteq Q$ for some abstract indexing $J$, and if $B \in Q$, where: ind $B=x$

$$
\begin{aligned}
& \&(\forall u)\left[u \in \operatorname{rel} B \Rightarrow \phi_{z}(u) \text { defined }\right] \\
& \&(\forall u)\left[u \in \operatorname{rel} B \Rightarrow \phi_{z}(u)=\text { ind } A_{\imath} \text { for some } \iota \in J\right] \\
& \&(\forall \bullet)\left[\iota \in J \Rightarrow \text { ind } A_{\imath}=\phi_{z}(u) \text { for some } u \in B\right] \\
& \&(\forall u)(\forall v)\left[\left\langle u, \imath^{\prime}\right\rangle \in \operatorname{rel} B \Rightarrow\left\langle\phi_{z}(u), \phi_{z}(v)\right\rangle \in \underset{\iota \in J}{\cup} \operatorname{rel} A_{\imath}\right] \\
& \&\left\langle x, \text { ind } A_{\iota_{0}}\right\rangle \in \underset{\imath \in J}{\cup} \operatorname{rel} A_{\imath} \text { for some } \iota_{0} \in J
\end{aligned}
$$


then $D \in Q$ where ind $D=3^{x} 5^{z}$ and $\operatorname{rel} D$ is the intersection of all binary relations $R$ such that $\left(\forall_{\iota}\right)\left[\iota \in J \Rightarrow \mathfrak{e}\left(A_{\iota}, R\right)\right]$.

As we shall see, the members of $R$ will be the "number classes" of the ultimate system.

Lemma 7. For any $A \in R$, the following are equivalent:

(i) $(\exists y)[\langle x, y\rangle \in \operatorname{rel} A]$;

(ii) $x=1$ or $(\exists y)[\langle y, x\rangle \in \operatorname{rel} A]$;

(iii) $x=1$ or $\langle 1, x\rangle \in \operatorname{rel} A$;

(iv) $\left\langle x, 2^{x}\right\rangle \in \operatorname{rel} A$.

Proof. (iv) $\Rightarrow$ (i) is immediate.

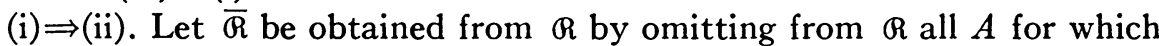
(i) $\Rightarrow$ (ii) fails. We show $\bar{R}$ is closed under conditions (1)-(3) of the definition of $R$. Hence $\bar{R}=R$. (Details are in $[\mathrm{Kr}]$.)

(ii) $\Rightarrow$ (iii) and (iii) $\Rightarrow$ (iv) are proved similarly. $y, z]$.

Lemma 8. $A \in R \& x \in A \Rightarrow\left[x=1\right.$ or $x=2^{y}$ for some $y$ or $x=3^{3} 5^{z}$ for some

Lemma 9. $A \in \mathbb{R} \Rightarrow(\forall x)[\langle x, 1\rangle \notin$ rel $A]$.

Lemma 10. If $A \in \mathbb{R}$, then $\left\langle u, 2^{x}\right\rangle \in \operatorname{rel} A \Rightarrow\langle u, x\rangle \in \operatorname{rel} A$ or $u=x$.

Lemma 11. If $A \in \mathcal{R}$, then $\langle u, v\rangle \in \operatorname{rel} A \Rightarrow\left[\left\langle 2^{u}, v\right\rangle \in \operatorname{rel} A\right.$ or $\left.2^{u}=v\right]$.

Proofs of Lemmas 8 through 11 are similar to the proof indicated for Lemma 7.

Lemma 12. If $A \in R$, and $x \neq 0$, then $x \in \operatorname{rel} A \Leftrightarrow 2^{x} \in \operatorname{rel} A$.

Proof. Corollary to Lemmas 7, 10 and 11.

Lemma 13. $A, B \in R$ and $A \neq B \Rightarrow$ ind $A \neq$ ind $B$.

Proof. Similar to Lemma 7.

Thus $R$ is countably infinite, and for $A$ in $R$ and $x=i n d A$, we can uniquely define:

Definition. $I_{x}={ }_{d f} r e l A$.

Lemma 14. (i) $A \in R$ \& ind $A=2^{x} \Rightarrow$ there exists $B \in R$ such that ind $B=x$ and $A$ is obtained from $B$ by (2) of the definition of $R$.

(ii) $A \in R \&$ ind $A=3^{x} 5^{2} \Rightarrow$ there exist $B \in R$ and $\left\{A_{\imath}\right\} \subseteq R$ such that $A$ is obtained from $\left\{A_{\imath}\right\}$ and $B$ by (3) of the definition of $R$.

Proof. Immediate.

LeMma 15. (i) $\left\langle m, 3^{a} 5^{b}\right\rangle \in I_{2^{x}} \Rightarrow\left[\left[a=x\right.\right.$ or $\left.\langle a, x\rangle \in I_{x}\right] \&(\exists v)\left[v \in r \ln ^{I_{x}} a\right.$ $\left.\left.\&\left\langle m, \phi_{b}(v)\right\rangle \in I_{2^{x}}\right]\right]$. 
(ii) $\left\langle m, 3^{a} 5^{b}\right\rangle \in I_{3^{y} b^{z}} \Rightarrow(\exists u)(\exists v)\left[u \in I_{\nu} \quad \&\left[a=\phi_{z}(u)\right.\right.$ or $\left.\left\langle a, \phi_{z}(u)\right\rangle \in I_{\phi_{z}(u)}\right]$ $\left.\& v \in r \ln ^{1} \phi_{z}(u) a \&\left\langle m, \phi_{b}(v)\right\rangle \in I_{3^{2} 5^{z}}\right]$.

Proof. Similar to that for Lemma 7.

Lemma 16. $A \in R \Rightarrow$ rel $A$ is transitive.

Proof. Immediate from definition of $\mathfrak{e}(A, R)$.

Definition. $\phi_{z}$ maps $R$ order-preserving into $S \equiv_{d f}(\forall u)\left[u \in R \Rightarrow \phi_{z}(u)\right.$ defined $] \&(\forall u)(\forall v)\left[\langle u, v\rangle \in R \Rightarrow\left\langle\phi_{z}(u), \phi_{z}(v)\right\rangle \in S\right]$.

That $R$ and relations $I_{x}$ in $R$ have the desired properties now appears in a main lemma.

LEmma 17. If $A \in R$, then

(i) rel $A$ is a well-ordered partial ordering,

(ii) ind $A \in \operatorname{rel} A$,

(iii) $3^{y} 5^{z} \in \operatorname{rel} A \Rightarrow\langle y$, ind $A\rangle \in \operatorname{rel} A$,

(iv) $\langle y$, ind $A\rangle \in \operatorname{rel} A \Rightarrow(\exists B)\left[B \in R \&\right.$ ind $\left.B=y \& \operatorname{rel} B=r \ln ^{A} y\right]$,

(v) $3^{y} 5^{z} \in$ rel $A \Rightarrow \phi_{z}$ maps $I_{y}$ order-preserving into rel $A \&(\exists u)\left[u \in I_{y}\right.$ $\left.\&\left\langle y, \phi_{z}(u)\right\rangle \in \operatorname{rel} A\right]$,

(vi) $\left\langle m, 3^{y} 5^{z}\right\rangle \in \operatorname{rel} A \Rightarrow(\exists u)\left[u \in I_{y} \&\left\langle m, \phi_{z}(u)\right\rangle \in \operatorname{rel} A\right]$,

(vii) $\langle y$, ind $A\rangle \in \operatorname{rel} A \& \phi_{z}$ maps $I_{y}$ order-preserving into rel $A$ $\&(\exists u)\left[u \in I_{y} \&\left\langle y, \phi_{z}(u)\right\rangle \in \operatorname{rel} A\right] \Rightarrow(\forall v)\left[v \in I_{y} \Rightarrow\left\langle\phi_{z}(v), 3^{y} 5^{z}\right\rangle \in \operatorname{rel} A\right]$.

Proof. Use the preceding lemmas and make a simultaneous application of the method of the proof of Lemma 7.

Lemma 18. $A, B \in R \Rightarrow \operatorname{rel} A$ and rel $B$ are coherent (in the sense of $\S 4$ ).

Proof. Use well-ordering given by Lemma 17.

Definition. $<\tilde{C}={ }_{d f} \bigcup_{A \in \mathbb{R}} \operatorname{rel} A ; \tilde{C}={ }_{d f} d o m<\tilde{c}$.

The above lemmas can now be used to give brief and direct proofs of the following theorems and corollary.

TheOREM $6 .<_{\tilde{C}}$ is a system of notations.

THEOREM 7. (i) $3^{a} 5^{b} \in \tilde{C} \Rightarrow a<\tilde{c} 3^{a} 5^{b}$,

(ii) $\widetilde{C}=\{x \mid x=$ ind $A$ for some $A \in \Re\}$.

Corollary. For each $x \in \tilde{C}, I_{x}$ is a system of notations.

Detailed proofs of Lemmas 7 through 18 and of Theorems 6 and 7 are found in [Kr, pp. 29-48].

Theorem 1 can now be applied to yield a segment of countable ordinal numbers for which $<\tilde{c}$ serves as system of notations. The analogy between $<\tilde{c}$ and the accessible ordinals is made clear by Lemma 17 and Theorem 7. The subsystems $I_{x}$ correspond to the various number classes of accessible ordinals. 
The relation between the segment of ordinal numbers covered by $<\tau$ and the segment covered by $<_{C}$ is an open question.

6. Expression of $C$ in both two-function-quantifier forms. In this section we discuss the system of notations $<_{c}$ obtained in $\$ 4$ in relation to the analytical hierarchy of Kleene $\left[\mathrm{K}_{2} ; \mathrm{K}_{3}\right]$. Specifically, we shall find analytical predicates in two-function-quantifier-universal and two-function-quantifierexistential forms respectively expressing the relation $<_{c}$. We recall that an analytical predicate is one that can be expressed explicitly in terms of constant and variable natural numbers, function variables, general recursive predicates, the connectives of propositional calculus, and the two types of quantifiers, number and function, each of two kinds, universal and existential (a predicate of this kind is called arithmetical if only number quantifiers are used). These predicates fall into a hierarchy according to the sequences of alternating function quantifiers required to define them from arithmetical predicates. More exactly, given an analytical predicate, the number and function quantifiers can be advanced by the usual rules of quantifiers to obtain a prenex form in which the scope of the prefix is recursive, and the prefix can be further reduced by applying one or several of the following alterationof-quantifiers rules listed in $\left[\mathrm{K}_{2}\right]$ :

(1) Adjacent number quantifiers of like kind can be contracted, since

$$
(\exists x)(\exists y) A(x, y) \equiv(\exists x) A\left((x)_{0},(x)_{1}\right),
$$

and

$$
(\forall x)(\forall y) A(x, y) \equiv(\forall x) A\left((x)_{0},(x)_{1}\right) .
$$

(2) Adjacent function quantifiers of like kind can be contracted, since

$$
(\exists \alpha)(\exists \beta) A(\alpha, \beta) \equiv(\exists \alpha) A\left(\lambda t(\alpha(t))_{0}, \lambda t(\alpha(t))_{1}\right)
$$

and

$$
(\forall \alpha)(\forall \beta) A(\alpha, \beta) \equiv(\forall \alpha) A\left(\lambda t(\alpha(t))_{0}, \lambda t(\alpha(t))_{1}\right) .
$$

(3) Number quantifiers can be raised in type to function quantifiers, since

$$
(\exists x) A(x) \equiv(\exists \alpha) A(\alpha(0)) \text {, }
$$

and

$$
(\forall x) A(x) \equiv(\forall \alpha) A(\alpha(0))
$$

Thus, in particular, adjacent quantifiers of mixed type and like kind can be contracted to a single function quantifier.

(4) A function quantifier can be advanced across a number quantifier of opposite kind, since

$$
(\exists x)(\forall \alpha) A(x, \alpha) \equiv(\forall \alpha)(\exists x) A\left(x, \lambda t \alpha\left(2^{x} 3^{\imath}\right)\right),
$$

and 


$$
(\forall x)(\exists \alpha) A(x, \alpha) \equiv(\exists \alpha)(\forall x) A\left(x, \lambda t \alpha\left(2^{x} 3^{t}\right)\right) .
$$

(5) A function quantifier applied directly to a recursive predicate can be reduced in type to a number quantifier, since, for example,

$$
(\exists \alpha) R(\alpha, a) \equiv(\exists x) T_{1}^{1}\left(\prod_{i<(x)_{0}} p_{i}^{(x)_{i+1}, f, a,(x)_{0}}\right)
$$

and

$$
(\forall \alpha) R(\alpha, a) \equiv(\forall x) \sim T_{1}^{1}\left(\prod_{i<(x)_{0}} p_{i}^{(x)_{i+1}, f, a,(x)_{0}}\right),
$$

where $T_{1}^{1}$ is defined as on page 291 of $\left[\mathrm{K}_{4}\right]$ and $f$ is a Gödel number depending on $R$. Similarly if $R$ has more free number and function variables.

By applying rules (1)-(5) any analytical predicate in $k$ free number variables $a_{1}, a_{2}, \cdots, a_{k}$ can be transformed to one of the forms

$$
\begin{aligned}
& (\forall \alpha)(\exists x) R\left(a_{1}, \cdots, a_{k}, \alpha, x\right), \quad(\exists \alpha)(\forall \beta)(\exists x) R(\cdots), \cdots \\
A\left(a_{1}, \cdots, a_{k}\right), & (\exists \alpha)(\forall x) R\left(a_{1}, \cdots, a_{k}, \alpha, x\right), \quad(\forall \alpha)(\exists \beta)(\forall x) R(\cdots), \cdots
\end{aligned}
$$

where $A$ is arithmetical and $R$ is general recursive. The same result holds if there are free function variables. Kleene's hierarchy theorem $\left[K_{2}, \S 4\right]$, states that for any of the above forms after the first, there is a predicate in that form which is not expressible in the dual form nor in any form with fewer quantifiers (although it is, of course, clear that predicates in any one of the forms can be expressed in any form with more quantifiers). We shall speak of the 1-function-quantifier-universal form, the 2-function-quantifier-universal form, the 2-function-quantifier-existential form [or more briefly the $1 F Q \forall$ form, the $2 F Q \forall$ form, the $2 F Q \exists$ form respectively], and so forth, when referring to the forms $(\forall \alpha)(\exists x) R(\cdots),(\forall \alpha)(\exists \beta)(\forall x) R(\cdots)$, $(\exists \alpha)(\forall \beta)(\exists x) R(\cdots), \cdots$.

An additional alteration-of-quantifiers rule is given by Addison and Kleene in $[\mathrm{AK}]$.

$$
(\forall \alpha)(\exists x)(\exists \beta) A(\bar{\alpha}(x), \beta) \equiv(\exists \beta)(\forall \alpha)(\exists x) A\left(\bar{\alpha}(x), \lambda t \beta\left(2^{\bar{\alpha}(x)} 3^{t}\right)\right),
$$

and

$$
(\exists \alpha)(\forall x)(\forall \beta) A(\bar{\alpha}(x), \beta) \equiv(\forall \beta)(\exists \alpha)(\forall x) A\left(\bar{\alpha}(x), \lambda t \beta\left(2^{\bar{\alpha}(x)} 3^{t}\right)\right) .
$$

It is interesting to note that in order to apply this rule it is only necessary that occurrences of $\alpha$ in the given predicate be restricted to expressions of the form $\bar{\alpha}(x)$. This is clearly not the case with the predicate

$$
(\forall \alpha)(\exists x)(\exists \beta)\left[(\forall y) \sim T_{1}^{\alpha, \beta}(a, a, y) \& x=x\right] .
$$


Many predicates, however, because of their particular structure, can be expressed in a form to which the rule is applicable. A large class of these is described in the following lemma. The statement of this lemma and of its corollary can be shortened; in the form in which they are given, however, the intended applications follow smoothly.

LeMma 19. Let $P$ be a predicate which is expressed explicitly in terms of constant and variable natural numbers, general recursive predicates, the function variable $\alpha$, the $l_{i}$-ary predicate variables $Q_{i}, 1 \leqq i \leqq r$, the connectives of propositional calculus, and number quantifiers. And let $Q_{i}^{0}, Q_{i}^{1}$ be predicates none of whose variables occur in $P$, with $l_{i}$ free number variables and no free function variables, and such that $Q_{i}^{0}$ can be transformed into $k F Q \forall$ form and $Q_{i}^{1}$ can be transformed into $k F Q \exists$ form by means of the quantifier rules listed above, $1 \leqq i \leqq r$, where $k \geqq 2$.

Then among the predicates $(\forall \alpha) P^{\prime}$, where $P^{\prime}$ is obtained from $P$ by replacing each occurrence $Q_{i}\left(u_{1}, \cdots, u_{l_{i}}\right)$ of $Q_{i}$ by either $Q_{i}^{0}\left(u_{1}, \cdots, u_{l_{i}}\right)$ or $Q_{i}^{1}\left(u_{1}, \cdots, u_{l_{i}}\right)$, there is a predicate $P_{1}$ transformable to $k F Q \forall$ form and a predicate $P_{2}$ transformable to $k F Q \exists$ form by means of the quantifier rules listed above.

Proof. We follow Kleene $\left[\mathrm{K}_{2}\right.$, p. 321] in calling an occurrence of $Q_{i}$ in $P$ positive or negative according as an initial quantifier at that occurrence would be unchanged or changed in kind when carried to the prefix of a prenex form for $P$.

Let $P_{1} \equiv(\forall \alpha) P^{\prime}$, where $P^{\prime}$ is obtained from $P$ by replacing each positive occurrence $Q_{i}\left(u_{1}, \cdots, u_{l_{i}}\right)$ of $Q_{i}$ by $Q_{i}^{0}\left(u_{1}, \cdots, u_{l_{i}}\right)$ and each negative occurrence $Q_{i}\left(u_{1}, \cdots, u_{l_{i}}\right)$ of $Q_{i}$ by $Q_{i}^{1}\left(u_{1}, \cdots, u_{l_{i}}\right)$. To obtain a $k F Q \forall$ expression for $P_{1}$ we first replace each occurrence of $Q_{i}^{0}$ in $P_{1}$ by a $k F Q \forall$ expression for it and each occurrence of $Q_{i}^{1}$ in $P_{1}$ by a $k F Q \exists$ expression for it, and then apply the quantifier rules (1)-(5) in a straightforward manner.

Let $P_{2} \equiv(\forall \alpha) P^{\prime \prime}$, where $P^{\prime \prime}$ is obtained from $P$ by replacing each positive occurrence $Q_{i}\left(u_{1}, \cdots, u_{l_{i}}\right)$ of $Q_{i}$ by $Q_{i}^{1}\left(u_{1}, \cdots, u_{l_{i}}\right)$ and each negative occurrence $Q_{i}\left(u_{1}, \cdots, u_{l_{i}}\right)$ of $Q_{i}$ by $Q_{i}^{0}\left(u_{1}, \cdots, u_{l_{i}}\right)$. To obtain a $k F Q \exists$ expression for $P_{2}$ we first replace each occurrence of $Q_{i}^{0}$ in $P_{2}$ by a $k F Q \forall$ expression for it with prefix $\left(\forall \beta_{1}\right)\left(\exists \beta_{2}\right) \cdots$, and each occurrence $Q_{i}^{1}$ in $P_{2}$ by a $k F Q \exists$ expression for it with prefix $\left(\exists \beta_{1}\right)\left(\forall \beta_{2}\right) \cdots$, and then advance all number quantifiers not in the scope of a function quantifier (i.e. the number quantifiers from $P$ ) to the front of $P^{\prime \prime}$ and use the quantifier rules (1)-(5) to obtain a prefix $(\forall \alpha)(\exists x)$ applied to a scope $B(\alpha, x)$. This scope $B(\alpha, x)$, as well as the scopes $C(\alpha, x)$ and $\bar{C}(\bar{\alpha}(x))$ mentioned below, may have other free number variables which we do not explicitly indicate. Now we make the following transformations:

(i) Replace each occurrence of $x$ in $B(\alpha, x)$ by $(x)_{0}$, thus obtaining the predicate $(\forall \alpha)(\exists x) B\left(\alpha,(x)_{0}\right)$ which is equivalent to $P_{2}$. 
(ii) Let $p_{1}, p_{2}, \cdots, p_{t}$ be all the terms in $B\left(\alpha,(x)_{0}\right)$ which occur as arguments of $\alpha$, and let $C(\alpha, x)$ be the predicate

$$
B\left(\alpha,(x)_{0}\right) \& p_{1}<x \& p_{2}<x \& \cdots \& p_{t}<x .
$$

Then since $\alpha$ has no occurrence in any of the $Q_{i}^{0}$ or $Q_{i}^{1}$, none of the expressions $p_{1}, \cdots, p_{t}$ contains a bound variable of $B\left(\alpha,(x)_{0}\right)$. Therefore the predicate $(\forall \alpha)(\exists x) C(\alpha, x)$ is equivalent to $P_{2}$.

(iii) Replace each occurrence $\alpha\left(p_{j}\right)$ of $\alpha$ in $C(\alpha, x)$ by $(\bar{\alpha}(x))_{p_{j}}, 1 \leqq j \leqq t$, and then replace each occurrence of $x$ not in the expression $\bar{\alpha}(x)$ by $\ln \bar{\alpha}(x)$ (cf. $\left.\left[K_{4}\right]\right)$. This results in a predicate $(\forall \alpha)(\exists x) \bar{C}(\bar{\alpha}(x))$ which is equivalent to $P_{2}$, and where $\bar{C}$ is expressed explicitly in ternıs of constant and variable natural numbers, general recursive predicates, the connectives of propositional calculus, and the $k$-function-quantifier expressions replacing $Q_{i}^{0}$ and $Q_{i}^{1}$.

Now the steps described above do not alter the positiveness or negativeness of an occurrence of one of the expressions replacing $Q_{i}^{0}$ or $Q_{i}^{1}$, hence in the number theoretic predicate $\bar{C}(s)$ obtained from $\bar{C}(\bar{\alpha}(x))$ by replacing $\bar{\alpha}(x)$ by the number variable $s$, each occurrence of a $k F Q \exists$ expression replacing $Q_{i}^{1}$ is positive and each occurrence of a $k F Q \forall$ expression replacing $Q_{i}^{0}$ is negative. Now advance the quantifiers $\left(\forall \beta_{1}\right)$ and $\left(\exists \beta_{1}\right)$, then $\left(\forall \beta_{2}\right)$ and $\left(\exists \beta_{2}\right), \cdots$, and finally $\left(\forall \beta_{k}\right)$ and $\left(\exists \beta_{k}\right)$ to the front of $\bar{C}(s)$ obtaining the prefix $\left(\exists \beta_{1}\right)\left(\forall \beta_{2}\right)\left(\exists \beta_{3}\right) \cdots\left(\beta_{k}\right)$ applied to an arithmetical predicate $A\left(s, \beta_{1}, \beta_{2}, \cdots, \beta_{k}\right)$. Thus we have the predicate

$$
(\forall \alpha)(\exists x)\left(\exists \beta_{1}\right)\left(\forall \beta_{2}\right) \cdots\left(\beta_{k}\right) A\left(\bar{\alpha}(x), \beta_{1}, \beta_{2}, \cdots, \beta_{k}\right)
$$

which is equivalent to $P_{2}$. But by quantifier rule (6) of Addison and Kleene, this predicate is equivalent to

$$
(\exists \beta)(\forall \alpha)(\exists x)\left(\forall \beta_{2}\right) \cdots\left(\beta_{k}\right) A\left(\bar{\alpha}(x), \lambda t \beta\left(2^{\bar{\alpha}(x)} 3^{t}\right), \beta_{2}, \cdots, \beta_{k}\right),
$$

and we can now apply the quantifier rules (1)-(5) to reduce the last predicate to the desired $k F Q \exists$ form. Q.E.D.

Corollary. Let $P$ be as in the lemma except that it has no free function variables. Let $Q_{i}^{0}, Q_{i}^{1}$ be as in the lemma, except that $k$ may also equal 1. Then among the predicates $P^{\prime}$ obtained from $P$ by replacing each occurrence $Q_{i}\left(u_{1}, \cdots, u_{l_{i}}\right)$ of $Q_{i}$ by either $Q_{i}^{0}\left(u_{1}, \cdots, u_{l_{i}}\right)$ or $Q_{i}^{1}\left(u_{1}, \cdots, u_{l_{i}}\right)$, there is a predicate $P_{1}$ transformable to $k F Q \forall$ form and a predicate $P_{2}$ transformable to $k F Q \exists$ form by means of the quantifier rules listed above.

Investigations by Kleene, Spector and others, of various systems of notations for the constructive ordinals have depended strongly on applications of the recursion theorem. The role played by the recursion theorem in these investigations has been summarized by Rogers [R] in a lemma concerning the defining of partial recursive functions over well-ordered partial orderings. We state this lemma here. 
$\phi_{x}$ is the partial recursive function of index $x$. Let $<_{w}$ be a well-ordered partial ordering over a set of non-negative integers $W . S$ is a segment of $W$ if $S \subseteq W$ and if $\left(a \in S \& b<_{w} a\right) \Rightarrow b \in S$. For $a \in W, S_{a}$ shall be the segment $\left\{b \mid b<_{w} a\right\}$; and $S_{a}^{*}$ shall be the segment $\left\{b \mid b \leqq_{w} a\right\}$.

Lemma 20. Let $<_{W}$ be a well-ordered partial ordering on $W$. Let $\mathfrak{D}(x, S)$ be a relation between integers $x$ and segments $S$ of $W$ such that

(i) $\mathfrak{D}(x, S)$ and $\phi_{x}=\phi_{y}$ on $S \Rightarrow \mathfrak{D}(y, S)$,

(ii) $\mathfrak{D}\left(x, S_{a}^{*}\right)$ for all $a \in S \Rightarrow \mathfrak{D}(x, S)$.

Let $f$ be a recursive function of two variables such that

(iii) $\mathfrak{D}\left(x, S_{a}\right) \Rightarrow\left[\mathfrak{D}\left(f(x, a), S_{a}^{*}\right)\right.$ and $\phi_{f(x, a)}=\phi_{x}$ on $\left.S_{a}\right]$.

Then there exists an integer $x_{0}$ such that $\mathfrak{D}\left(x_{0}, W\right)$.

The lemma says, in effect, that given a property $\pi$ of certain members of $W$, if a uniform effective procedure for showing that all members of $S_{a}^{*}$ have $\pi$ can always be obtained from a uniform effective procedure for showing that all members of $S_{a}$ have $\pi$, then there is a uniform effective procedure for showing that all members of $W$ have $\pi$. In dealing with constructive systems of notation for ordinal numbers, therefore, the recursion theorem assumes a role similar to that played by transfinite induction in the classical theory of ordinal numbers.

In our applications of this lemma, given a relation $\mathfrak{D}(x, \mathrm{~S})$ satisfying (i) and (ii), our main task is to define the recursive function $f$ in such a way that (iii) is satisfied. The definition of $f$ will, in general, depend on somewhat deeper structural properties of $<_{w}$.

We return now to the problem of finding $2 F Q \forall$ and $2 F Q \exists$ expressions for $<_{c}$. From the definition of $\lesssim_{c}$ one can obtain directly a predicate in $2 F Q \forall$ form expressing $\lesssim_{C}\left({ }^{10}\right)$, but there seems to be no simple way to obtain a $2 F Q \exists$ form expressing $\Sigma_{c}$ without going more deeply into the structure of $\lesssim_{c}$ and the chain $D$ of relations defined in $\S 4\left({ }^{11}\right)$. In Theorems 8,9 below, we proceed inductively by way of $D$ to obtain both the $2 F Q \forall$ and the $2 F Q \exists$ forms.

Definition. For $a \in C$, let $D_{a}$ be the least relation in $D$ whose domain

(10) To thus obtain this $2 F Q$ form observe, from the results of $\S 4$, that $x \lesssim c y$ can be equivalently written: $(\forall R)[[R$ transitive, reflexive and connected \& $R$ well-ordered \& $(\forall S)$ [S initial segment of $R \Leftrightarrow f^{*}(S)$ initial segment of $\left.R\right] \&\{\langle 1,1\rangle\}$ an initial segment of $R$ ] $\Longrightarrow\langle x, y\rangle \in R]$. Going over to characteristic functions and recursive predicates, we observe that function quantifiers appear in the positions: $(\forall \alpha)[[\cdots \&(\forall \beta) \cdots \&(\forall \gamma)[\cdots \Leftrightarrow \cdots]$ $\& \cdots] \Rightarrow \cdots$ ] yielding the $2 F Q \forall$ form.

(11) Unfortunately, the Addison-Kleene quantifier rule appears not to apply directly to the $2 F Q \forall$ form suggested in footnote 10. In particular, the $R$ and $S$ mentioned there may be infinite, in which case a relationship such as " $S$ initial segment of $R$ " involves a more complex corresponding relationship between the representing functions $\alpha$ and $\gamma$ than can be immediately subsumed under the Addison-Kleene rule. 
contains $a$. For $R \in \mathcal{D}$, let $R^{-}$be the relation $P(R)$.

We first show that each $D_{a}, a \in C$, is expressible in both $2 F Q$ forms.

THEOREM 8. There exists a recursive function $\theta$ such that for any $a \in C$,

$$
\begin{aligned}
\langle m, n\rangle \in D_{a} & \equiv(\forall \alpha)(\exists \beta)(\forall y) \sim T_{2}^{\alpha, \beta}\left((\theta(a))_{0}, m, n, y\right) \\
& \equiv(\exists \alpha)(\forall \beta)(\exists y) T_{2}^{\alpha, \beta}\left((\theta(a))_{1}, m, n, y\right) .
\end{aligned}
$$

Proof. The proof will consist of an application of Lemma 20 to the wellordered partial ordering $<_{c}$. The relation $\mathfrak{D}(x, S)$ of Lemma 20 is defined for an integer $x$ and segment $S$ of $C$ by:

$\mathfrak{D}(x, S) \equiv\left\{\phi_{x}\right.$ is defined on $S$, and for each $b \in S,\left(\phi_{x}(b)\right)_{0}$ and $\left(\phi_{x}(b)\right)_{1}$ are indices for $D_{b}$ in $2 F Q \forall$ and $2 F Q \exists$ forms respectively [I.e. $\langle m, n\rangle \in D_{, b} \equiv(\forall \alpha)(\exists \beta)(\forall y) \sim T_{2}^{\alpha, \beta}\left(\left(\phi_{x}(b)\right)_{0}, m, n, y\right)$ $\left.\left.\equiv(\exists \alpha)(\forall \beta)(\exists y) T_{2}^{\alpha, \beta}\left(\left(\phi_{x}(b)\right)_{1}, m, n, y\right).\right]\right\}$.

Note that conditions (i) and (ii) of Lemma 20 are trivially satisfied. We shall define below the recursive function $f(x, a)$ required in the application of the lemma. The desired recursive function $\theta$ is then $\theta=\phi_{x_{0}}$, where $x_{0}$ is an integer such that $\mathfrak{D}\left(x_{0}, C\right)$.

Define the partial recursive function $\phi_{e}(x, a, t)$ by

$$
\phi_{e}(x, a, t)= \begin{cases}2^{e_{1} 3^{e 2}} & \text { if } t=a, \\ \phi_{x}(t) & \text { if } t \neq a,\end{cases}
$$

where $e_{1}$ and $e_{2}$ depend effectively on $x$ and $a$ and will be defined below in Cases 1-4, and let

$$
f(x, a)=S_{1}^{2}(e, x, a) .
$$

We shall see that $e_{1}$ and $e_{2}$ are defined for all $a$ and $x$; it follows that $\phi_{f(x, a)}(a)$ is always defined, and hence, from the proof of Lemma 20 in [R], that $\theta=\phi_{x_{0}}$ is everywhere defined.

For $a$ in $C, e_{1}$ and $e_{2}$ are to be Gödel numbers for $D_{a}$ in $2 F Q \forall$ and $2 F Q \exists$ forms respectively. Our general attack will be as follows. In order to satisfy condition (iii) of Lemma 20, we must show how, given an effective function that provides the $2 F Q$ forms for all $D_{b}, b<_{c} a$, to obtain the $2 F Q$ forms for $D_{a}$. Before giving full formal details we make some comments.

In case $a=1$, the construction of $e_{1}$ and $e_{2}$ will be immediate.

In case $a \in C \& a=2^{b} \& b \neq 0$, the construction will follow from an application of Lemma 19 to the identity

$$
\begin{array}{r}
\langle m, n\rangle \in D_{a} \equiv\langle m, n\rangle \in D_{b} \vee(\exists t)\left[n=2^{t} \&\langle m, t\rangle \in D_{b}\right] \\
\vee(\exists s)(\exists t)\left[m=2^{\bullet} \& n=2^{t} \&\langle s, t\rangle \in D_{b}\right] .
\end{array}
$$

This identity follows from $\left(1^{*}\right)$ of $\$ 4$. 
In case $a \in C \& a=3^{b} 5^{z}$, the construction will occur in two stages. First the $2 F Q$ forms for $D_{3^{b} b^{i}}^{-}$are obtained; and then the $2 F Q$ forms for $D_{3^{b} b^{2}}$ are obtained from these.

The first stage will use, indirectly, the identities

$$
\begin{aligned}
& D_{1} \subseteq D_{3^{b} 5^{i}} \\
& (\forall t)\left[t \in D_{3^{b} 5^{i}} \Rightarrow D_{2^{t}} \subseteq D_{3^{b} 5^{i}}\right] \text {; } \\
& (\forall y)\left[y \in D_{b}^{-} \Rightarrow D_{3^{y_{5}}} \subseteq D_{3^{b} 5^{i}}\right] \text {; } \\
& (\forall y)(\forall e)\left[y \in \overline{D_{b}^{-}} \&(\forall u)\left[u \in \overline{D_{3^{y} b^{i}}} \Rightarrow \phi_{e}(u) \text { defined \& } \phi_{e}(u) \in D_{3^{b_{5}}}\right]\right.
\end{aligned}
$$

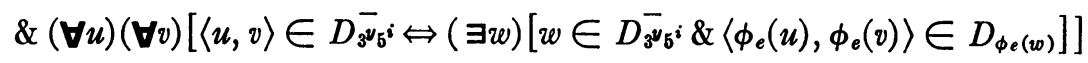

$$
\begin{aligned}
& \left.\Rightarrow D_{3^{y_{5}}} \subseteq D_{3^{b} 5^{i}}\right] \text {. }
\end{aligned}
$$

The four parts of $\left(\mathrm{Id}_{2}\right)$ are justified by Lemma 6 in $\$ 4$.

For the second stage we use the identities $\left(\operatorname{Id}_{3}\right)$ and $\left(\operatorname{Id}_{4}\right)$, to be given below in course of the construction. $\left(\mathrm{Id}_{3}\right)$ defines $D_{3^{b} b^{z}}^{-}$in terms of $D_{3^{b} b^{i}}^{-}$, and $\left(\mathrm{Id}_{4}\right)$ defines $D_{3^{b} 5^{z}}$ in terms of $D_{3^{b} 5^{z}}^{\bar{a}}$ and various $D_{a}, a<_{c} 3^{b} 5^{z}$. These identities follow from Lemma 6 and from $\left(2^{*}\right)$ and $\left(3^{*}\right)$ of $\$ 4$.

We next give the construction and then go on to prove that it gives the desired $2 F Q$ forms.

CASE 1. $a=1$ : Let $e_{1}$ and $e_{2}$ define recursively from $\alpha$ and $\beta$ the partial functions

$$
\lambda m n \mu y[\sim(m=n=1) \& y=0]
$$

and

$$
\lambda m n \mu y[m=n=1 \& y=0]
$$

respectively.

CASE 2. $a=2^{b} \& b \neq 0$ : Let $Q$ be a predicate letter and consider the predicate $P$ defined by

$$
\begin{gathered}
P(x, a, m, n) \equiv Q(x, b, m, n) \vee(\exists t)\left[n=2^{t} \& Q(x, b, m, t)\right] \\
\vee(\exists s)(\exists t)\left[m=2^{s} \& n=2^{t} \& Q(x, b, s, t)\right] .
\end{gathered}
$$

(Motivation: $\mathrm{cf} .\left(\mathrm{Id}_{1}\right)$ above.)

Applying the corollary to Lemma 19 with $r=1, k=2$,

$$
Q^{0}\left(y_{1}, y_{2}, y_{3}, y_{4}\right) \equiv\left[\phi_{y_{1}}\left(y_{2}\right)\right. \text { is defined and }
$$

$$
\left.(\forall \alpha)(\exists \beta)(\forall y) \sim T_{2}^{\alpha, \beta}\left(\left(\phi_{y_{1}}\left(y_{2}\right)\right)_{0}, y_{3}, y_{4}, y\right)\right],
$$

and

$$
\begin{aligned}
Q^{1}\left(y_{1}, y_{2}, y_{3}, y_{4}\right) \equiv[ & \phi_{\nu_{1}}\left(y_{2}\right) \text { is defined and } \\
& \left.(\exists \alpha)(\forall \beta)(\exists y) T_{2}^{\alpha, \beta}\left(\left(\phi_{y_{1}}\left(y_{2}\right)\right)_{1}, y_{3}, y_{4}, y\right)\right],
\end{aligned}
$$


we obtain predicates $P_{1}$ and $P_{2}$ such that

$$
P_{1}(x, a, m, n) \equiv(\forall \dot{\alpha})(\exists \beta)(\forall y) \sim T_{4}^{\alpha, \beta}\left(p_{1}, x, a, m, n, y\right)
$$

and

$$
P_{2}(x, a, m, n) \equiv(\exists \alpha)(\forall \beta)(\exists y) T_{4}^{\alpha, \beta}\left(p_{2}, x, a, m, n, y\right)
$$

for some fixed integers $p_{1}$ and $p_{2}$. Now let $e_{i}^{\prime}$ define recursively from $\alpha$ and $\beta$ the partial function

$$
\lambda p_{i} x a m n \mu y T_{4}^{\alpha, \beta}\left(p_{i}, x, a, m, n, y\right)
$$

and let

$$
e_{i}=S_{2}^{3,1,1}\left(e_{i}^{\prime}, p_{i}, x, a\right)
$$

for $i=1,2$.

CASE 3. $a=3^{b} 5^{z}$ : Let $i$ be a fixed index of the identity function and $Q$ a predicate letter, and consider the predicate $P$ defined by

$\left(^{*}\right) P(x, \quad s, \quad m, \quad n) \equiv(\forall \alpha) \cdot\{\alpha(1, \quad 1)=0 \&(\forall t)[(\exists q)(\alpha(t, \quad q)=0)$ $\left.\Rightarrow(\forall u)(\forall v)\left(Q\left(x, 2^{t}, u, v\right) \Rightarrow \alpha(u, v)=0\right)\right] \&(\forall y)[Q(x, s, y, s) \& \sim Q(x, s, s, y)$ $\left.\Rightarrow(\forall u)(\forall v)\left(Q\left(x, 3^{y} 5^{i}, u, v\right) \Rightarrow \alpha(u, v)=0\right)\right] \&(\forall y)(\forall e)[Q(x, s, y, s)$ $\& \sim Q(x, s, s, y) \&(\forall u)\left[(\exists q)\left(Q\left(x, 3^{y} 5^{i}, u, q\right) \& \sim Q\left(x, 3^{y} 5^{i}, q, u\right)\right) \Rightarrow \phi_{e}(u)\right.$ is defined \& $\left.(\exists q)\left(\alpha\left(\phi_{e}(u), q\right)=0\right)\right] \quad \& \quad(\forall u)(\forall v)\left[Q\left(x, \quad 3^{y} 5^{i}, \quad u, \quad v\right)\right.$ $\&(\exists q)\left(\sim Q\left(x, 3^{y} 5^{i}, q, v\right)\right) \Leftrightarrow(\exists w)\left((\exists q)\left(Q\left(x, 3^{y} 5^{i}, w, q\right) \& \sim Q\left(x, 3^{y} 5^{i}, q, w\right)\right)\right.$ $\left.\left.\left.\left.\& Q\left(x, \phi_{e}(w), \phi_{e}(u), \phi_{e}(v)\right)\right)\right] \Rightarrow(\forall u)(\forall v)\left[Q\left(x, 3^{y} 5^{e}, u, v\right) \Rightarrow \alpha(u, v)=0\right]\right]\right\}$ $\Rightarrow \alpha(m, n)=0$.

(Motivation: cf. $\left(\mathrm{Id}_{4}\right)$, recalling that $\left[n \in D_{a}^{-} \equiv\langle n, a\rangle \in D_{a} \&\langle a, n\rangle \notin D_{a}\right]$ and $\left[\langle m, n\rangle \in D_{a}^{-} \equiv\langle a, n\rangle \in D_{a} \&\langle m, n\rangle \in D_{a}\right]$, and reading " $\langle v, w\rangle \in D_{u}$ " for $Q(x, u, v, w)$ and " $\langle m, n\rangle \in D_{3^{*} i^{i}}^{-}$" for $P(x, s, m, n)$. Use of $\left(^{*}\right)$ and role of " $\forall \alpha)$ " will be explained and justified below.)

Applying Lemma 19 with $r, k, Q^{0}, Q^{1}$ as in Case 2, we obtain predicates $P_{1}^{\prime}$ and $P_{2}^{\prime}$ such that

$$
P_{1}^{\prime}(x, s, m, n) \equiv(\forall \alpha)(\exists \beta)(\forall y) \sim T_{4}^{\alpha, \beta}\left(p_{1}^{\prime}, x, s, m, n, y\right)
$$

and

$$
P_{2}^{\prime}(x, s, m, n) \equiv(\exists \alpha)(\forall \beta)(\exists y) T_{4}^{\alpha, \beta}\left(p_{2}^{\prime}, x, s, m, n, y\right)
$$

for some fixed integers $p_{1}^{\prime}$ and $p_{2}^{\prime}$.

Now if $3^{b} 5^{z} \in C, D_{3^{b} 5^{z}}$ is definable explicitly from $D_{3^{b} 5^{i}}$ as follows:

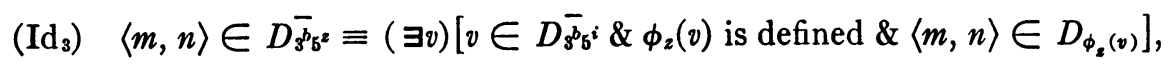
and 


$$
\begin{aligned}
& \langle m, n\rangle \in D_{3^{b} 5^{s}} \equiv\langle m, n\rangle \in \overline{D_{3^{b} 5^{s}}}
\end{aligned}
$$

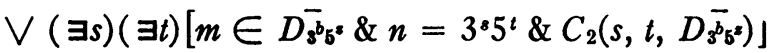

$$
\begin{aligned}
& \vee\left(\exists s_{1}\right)\left(\exists t_{1}\right)\left(\exists s_{2}\right)\left(\exists t_{2}\right)\left[m=3^{s_{1}} 5^{t_{1}} \& n=3^{s_{2}} 5^{t_{2}}\right.
\end{aligned}
$$

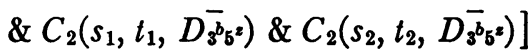

$$
\begin{aligned}
& \vee\left\{( \forall s ) ( \forall t ) \left[(\exists w)\left(3^{8} 5^{w} \in \overline{D_{3^{b} 5^{3}}}\right) \& O P\left(t, \overline{D_{3^{7} 5^{i}}}, \overline{D_{8^{b} 5^{z}}}\right)\right.\right. \\
& \left.\Rightarrow \sim O P C\left(t, \overline{D_{3^{5} 5^{i}}}, \overline{D_{s^{b}} 5^{3}}\right)\right]
\end{aligned}
$$

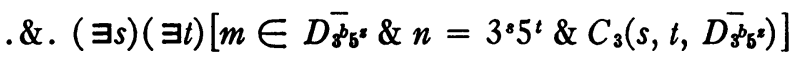

$$
\begin{aligned}
& \bigvee\left(\exists s_{1}\right)\left(\exists t_{1}\right)\left(\exists s_{2}\right)\left(\exists t_{2}\right)\left[m=3^{s_{1}} 5^{t_{1}} \& n=3^{\theta_{2}} 5^{t_{2}}\right. \\
& \left.\left.\& C_{3}\left(s_{1}, t_{1}, \overline{D_{3}^{6} 5^{z}}\right) \& C_{3}\left(s_{2}, t_{2}, \overline{D_{3^{b}} b^{2}}\right)\right]\right\} \text {, }
\end{aligned}
$$

where $O P, O P C, C_{2}, C_{3}$, are as defined in $\S 4$.

Now let $P^{\prime \prime}(x, a, m, n)$ be the predicate obtained from the above expression for $\langle m, n\rangle \in D_{3^{b} b^{3}}$ by replacing

(1) $O P, O P C, C_{2}, C_{3}$, by the expressions which they abbreviate,

(2) rel $\bar{D}_{\bar{b}_{5} s} y$ by $D_{3^{y_{5}}}^{-}$

(3) expressions of the form $x \in R$ by $(\exists r)[\langle x, r\rangle \in R]$, $\left(y=s, s_{1}, s_{2}\right)$,

(4) expressions of the form $\left\langle y_{1}, y_{2}\right\rangle \in D_{3^{b} 5^{z}}^{-}$by the expanded expression of $\left(\mathrm{Id}_{3}\right)$,

(5) expressions of the form $\left\langle y_{1}, y_{2}\right\rangle \in D_{3_{3} 5^{i}}^{-}$by $Q_{1}\left(x, y_{3}, y_{1}, y_{2}\right)$,

(6) expressions of the form $\left\langle y_{1}, y_{2}\right\rangle \in D_{y_{3}}$ by $\left[y_{3} \neq 1 \& Q_{2}\left(x, y_{3}, y_{1}, y_{2}\right)\right]$ $\vee\left[y_{3}=1 \& y_{2}=1 \& y_{1}=1\right]$, where $Q_{1}$ and $Q_{2}$ are predicate letters.

Then $P^{\prime \prime}$ is arithmetical in $Q_{1}$ and $Q_{2}$. Therefore applying the corollary to Lemma 19 with $r=2, k=2$,

$$
\begin{aligned}
& Q_{1}^{0}\left(y_{1}, y_{2}, y_{3}, y_{4}\right) \equiv P_{1}^{\prime}\left(y_{1}, y_{2}, y_{3}, y_{4}\right), \\
& Q_{1}^{1}\left(y_{1}, y_{2}, y_{3}, y_{4}\right) \equiv P_{2}^{\prime}\left(y_{1}, y_{2}, y_{3}, y_{4}\right), \\
& Q_{2}^{0}=Q^{0} \text { of Case } 2,
\end{aligned}
$$

and

$$
Q_{2}^{1}=Q^{1} \text { of Case 2, }
$$

we obtain predicates $P_{1}^{\prime \prime}$ and $P_{2}^{\prime \prime}$ such that

$$
P_{1}^{\prime \prime}(x, a, m, n) \equiv(\forall \alpha)(\exists \beta)(\forall y) \sim T_{1}^{\alpha, \beta}\left(p_{1}^{\prime \prime}, x, a, m, n, y\right)
$$

and

$$
P_{2}^{\prime \prime}(x, a, m, n) \equiv(\exists \alpha)(\forall \beta)(\exists y) T_{1}^{\alpha, \beta}\left(p_{2}^{\prime \prime}, x, a, m, n, y\right)
$$

for some fixed integers $p_{1}^{\prime \prime}$ and $p_{2}^{\prime \prime}$. 
Now let $e_{i}^{\prime \prime}$ define recursively from $\alpha$ and $\beta$ the partial function

$$
\lambda p_{i}^{\prime \prime} x a m n \mu y T_{4}^{\alpha, \beta}\left(p_{i}^{\prime \prime}, x, a, m, n, y\right),
$$

and let

$$
e_{i}=S_{2}^{3,1,1}\left(e_{i}^{\prime \prime}, p_{i}^{\prime \prime}, x, a\right)
$$

for $i=1,2$.

CASE 4. Otherwise let $e_{1}=e_{2}=0$.

This completes the definition of $e_{1}$ and $e_{2}$ and hence of $f(x, a)$. We complete the proof of the theorem by showing that $f(x, a)$ satisfies condition (iii) of Lemma 20.

Let $a \in C$ and let $x$ be an integer such that $\mathfrak{D}\left(x, S_{a}\right)$. By definition of $f(x, a), \phi_{f(x, a)}(t)=\phi_{x}(t)$ on $S_{a}$. Hence we need only show that $\mathfrak{D}\left(f(x, a), S_{a}^{*}\right)$; i.e. we must show that $\left(\phi_{f(x, a)}(a)\right)_{0}=e_{1}$ and $\left(\phi_{f(x, a)}(a)\right)_{1}=e_{2}$ are indices for $D_{a}$ in $2 F Q \forall$ and $2 F Q \exists$ forms respectively. We prove this by cases corresponding to the definition of $e_{1}$ and $e_{2}$.

CASE $1^{\prime} . a=1:(\forall \alpha)(\exists \beta)(\forall y) \sim T_{2}^{\alpha, \beta}\left(e_{1}, m, n, y\right) \equiv$ $(\exists \alpha)(\forall \beta)(\exists y) T_{2}^{\alpha, \beta}\left(e_{2}, m, n, y\right) \equiv m=n=1 \equiv\langle m, n\rangle \in D_{1}$.

CASE $2^{\prime} . a=2^{b} \& b \neq 0$ : Since $\left(\phi_{x}(b)\right)_{0}$ and $\left(\phi_{x}(b)\right)_{1}$ are indices for $D_{b}$ in $2 F Q \forall$ and $2 F Q \exists$ forms we have

$$
Q^{0}\left(x, b, y_{1}, y_{2}\right) \equiv Q^{1}\left(x, b, y_{1}, y_{2}\right) \equiv\left\langle y_{1}, y_{2}\right\rangle \in D_{b} .
$$

Therefore

$$
\begin{aligned}
P_{1}(x, a, m, n) & \equiv P_{2}(x, a, m, n) \\
& \equiv\langle m, n\rangle \in D_{b} \bigvee(\exists t)\left[n=2^{t} \&\langle m, t\rangle \in D_{b}\right] \\
& \vee(\exists s)(\exists t)\left[m=2^{s} \& n=2^{t} \&\langle s, t\rangle \in D_{b}\right] \\
& \equiv\langle m, n\rangle \in D_{a} .
\end{aligned}
$$

Thus by definition of $e_{1}$ and $e_{2}$,

$$
\begin{aligned}
(\forall \alpha)(\exists \beta)(\forall y) & \sim T_{2}^{\alpha, \beta}\left(e_{1}, m, n, y\right) \\
& \equiv(\exists \alpha)(\forall \beta)(\exists y) T_{2}^{\alpha, \beta}\left(e_{2}, m, n, y\right) \\
& \equiv P_{1}(x, a, m, n) \equiv P_{2}(x, a, m, n) \\
& \equiv\langle m, n\rangle \in D_{a} .
\end{aligned}
$$

CASE $3^{\prime} . a=3^{b} 5^{2}$. We first prove a lemma which explains and justifies $\left({ }^{*}\right)$.

Lemma. Let $a \in C$ and let $R_{0}, R_{1}, \cdots$ be any sequence of binary relations on $N$ such that $R_{c}=D_{c}$ for all $c<_{c} a$. Then if $s<_{c} a$ and $s$ is an index in $S_{a}^{*}, D_{3^{e} 5^{i}}^{-}$ is the intersection $\tilde{S}$ of all binary relations $S$ on $N$ satisfying 
(a) $R_{1} \subseteq S$;

(b) $t \in S \Rightarrow R_{2}{ }^{t} \subseteq S$;

(c) $\langle y, s\rangle \in R_{8} \&\langle s, y\rangle \notin R_{s} \Rightarrow R_{3^{y} 5^{i}} \subseteq S$;

(d) $\langle y, \quad s\rangle \in R_{8} \&\langle s, \quad y\rangle \notin R_{8} \&(\forall u)\left[\left\langle u, \quad 3^{y} 5^{i}\right\rangle \in R_{3^{y} 5^{i}} \&\left\langle 3^{y} 5^{i}, \quad u\right\rangle \notin R_{3^{\prime} 5^{2}}\right.$ $\Rightarrow \phi_{e}(u)$ defined \& $\left.\phi_{e}(u) \in S\right] \&(\forall u)(\forall v)\left[\langle u, v\rangle \in R_{3^{y} 5^{i}} \&\left\langle 3^{y} 5^{i}, v\right\rangle \notin R_{3^{y} 5^{2}}\right.$ $\left.\Leftrightarrow(\exists w)\left[\left\langle w, 3^{y} 5^{i}\right\rangle \in R_{3^{y} 5^{i}} \&\left\langle 3^{y} 5^{i}, w\right\rangle \notin R_{3^{y} 5^{i}} \&\left\langle\phi_{e}(u), \phi_{e}(v)\right\rangle \in D_{\phi_{e}(w)}\right]\right] \Rightarrow R_{3^{y} 5^{e}}$ $\subseteq S$.

Proof of lemma. It is immediate from $\left(\operatorname{Id}_{2}\right)$ that $S=D_{3^{8} 5^{i}}^{-}$satisfies (a)-(d).

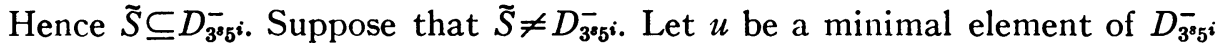
such that for some $v,\langle v, u\rangle \in D_{3^{8} 5^{i}}^{-}$but $\langle v, u\rangle \notin \tilde{S}$. It is clear that $\tilde{S}$ satisfies (a)-(d). Thus if $u=1$, then $v=1$ and hence $\langle 1,1\rangle \notin \tilde{S}$ which is a contradiction. If $u=2^{t}$, then $t \in \tilde{S}$, hence $D_{2}{ }^{t}=R_{2}{ }^{t} \subseteq \tilde{S}$. But then by coherence we have $\langle v, u\rangle \in D_{2}{ }^{t}$ and hence $\langle v, u\rangle \in \widetilde{S}$ contrary to the choice of $u$ and $v$. And if $u=3^{y} 5^{z}$, then $y<_{c} s$; hence $\langle y, s\rangle \in D_{s}=R_{s}$ and $\langle s, y\rangle \in D_{s}=R_{s}$. Then by (c), $D_{3^{y} 5^{i}} \subseteq \widetilde{S}$, and by (d), $D_{3^{y} 5^{z}} \subseteq \tilde{S}$. Hence since by coherence we have $\langle v, u\rangle \in D_{3^{y} 5^{z}}$, it follows that $\langle v, u\rangle \in \tilde{S}$ contrary to the choice of $u$ and $v$. We must therefore have $\widetilde{S}=D_{3^{3} 5^{i}}^{-}$. This proves the lemma.

Corollary. Let $a \in C$ and let $R_{0}^{\prime}, R_{1}^{\prime}, \cdots$ and $R_{0}^{\prime \prime}, R_{1}^{\prime \prime}, \cdots$ be any two sequences of binary relations on $N$ such that $R_{c}^{\prime}=R_{c}^{\prime \prime}=D_{c}$ for all $c<_{c}$ a. Then the conclusion of the lemma will remain true if some occurrences of $R$ are replaced by $R^{\prime}$ and the remaining occurrences of $R$ are replaced by $R^{\prime \prime}$ in its statement.

Now, by hypothesis, if $c<_{c} a, \lambda m n Q^{0}(x, c, m, n) \equiv \lambda m n Q^{1}(x, c, m, n)$ $\equiv\langle m, n\rangle \in D_{c} . P_{1}^{\prime}(x, s, m, n)$ is equivalent to the predicate obtained from $\left(^{*}\right)$ by replacing each occurrence of $Q$ by either $Q^{0}$ or $Q^{1}$. Hence applying the corollary to the lemma with

$$
R_{c}^{\prime}=\left\{\langle m, n\rangle \mid Q^{0}(x, c, m, n)\right\}
$$

and

$$
R_{c}^{\prime \prime}=\left\{\langle m, n\rangle \mid Q^{1}(x, c, m, n)\right\}
$$

we have

$$
\lambda m n P_{1}^{\prime}(x, s, m, n) \equiv\langle m, n\rangle \in \overline{D_{3^{8} 5^{i}}}
$$

It follows similarly that

$$
\lambda m n P_{2}^{\prime}(x, s, m, n) \equiv\langle m, n\rangle \in \overline{D_{3^{*} 5^{i}}} .
$$

Now by definition of $P^{\prime \prime}(x, a, m, n)$ and the method whereby $P_{1}^{\prime \prime}$ and $P_{2}^{\prime \prime}$ were obtained from $P^{\prime \prime}$ we see by $\left(\operatorname{Id}_{4}\right)$ that $\lambda m n P_{1}^{\prime \prime}(x, a, m, n)$ $\equiv \lambda m n P_{2}^{\prime \prime}(x, a, m, n) \equiv\langle m, n\rangle \in D_{3^{b} 5^{z}}=D_{a}$. By definition of $e_{1}$ and $e_{2}$, therefore, we have 


$$
\begin{aligned}
(\forall \alpha)(\exists \beta)(\forall y) & \sim T_{2}^{\alpha, \beta}\left(e_{1}, m, n, y\right) \\
& \equiv(\exists \alpha)(\forall \beta)(\exists y) T_{2}^{\alpha, \beta}\left(e_{2}, m, n, y\right) \\
& \equiv\langle m, n\rangle \in D_{a} .
\end{aligned}
$$

This completes the proof of the theorem. Q.E.D.

COROLlary 1. There exists a recursive function $\theta^{\prime}$ such that for any $a \in C$, $\left(\theta^{\prime}(a)\right)_{0}$ and $\left(\theta^{\prime}(a)\right)_{1}$ are indices for $D_{a}^{-}$in $2 F Q \forall$ and $2 F Q \exists$ forms respectively.

Proof. Since $\langle m, n\rangle \in D_{a}^{-} \equiv\langle m, n\rangle \in D_{a} \&\langle a, n\rangle \notin D_{a}$, we consider the predicate $P$ defined by

$$
P(a, m, n) \equiv Q(a, m, n) \& \sim Q(a, a, n) .
$$

Applying the corollary to Lemma 19 with $r=1, k=2$,

$$
Q^{0}\left(y_{1}, y_{2}, y_{3}\right) \equiv(\forall \alpha)(\exists \beta)(\forall y) \sim T_{2}^{\alpha, \beta}\left(\left(\theta\left(y_{1}\right)\right)_{0}, y_{2}, y_{3}, y\right)
$$

and

$$
Q^{1}\left(y_{1}, y_{2}, y_{3}\right) \equiv(\exists \alpha)(\forall \beta)(\exists y) T_{2}^{\alpha, \beta}\left(\left(\theta\left(y_{1}\right)\right)_{1}, y_{2}, y_{3}, y\right),
$$

we obtain predicates $P_{1}$ and $P_{2}$ such that

$$
P_{1}(a, m, n) \equiv(\forall \alpha)(\exists \beta)(\forall y) \sim T_{3}^{\alpha, \beta}\left(p_{1}, a, m, n, y\right)
$$

and

$$
P_{2}(a, m, n) \equiv(\exists \alpha)(\forall \beta)(\exists y) T_{3}^{\alpha, \beta}\left(p_{2}, a, m, n, y\right)
$$

for some fixed integers $p_{1}$ and $p_{2}$.

Letting $e_{i}^{\prime}$ define recursively from $\alpha$ and $\beta$ the function

$$
\lambda p_{i} a m n \mu y T_{3}^{\alpha, \beta}\left(p_{i}, a, m, n, y\right)
$$

for $i=1,2$, we put

$$
\theta^{\prime}(a)=2^{S_{2}^{2,1,1}}\left(e_{1}^{\prime}, p_{1}, a\right) 3^{S_{2}^{2,1,1}}\left(e_{2}^{\prime}, p_{2}, a\right) .
$$

COROLlaRY 2. There exists a recursive function $\theta^{\prime \prime}$ such that for any $a \in C$, $\left(\theta^{\prime \prime}(a)\right)_{0}$ and $\left(\theta^{\prime \prime}(a)\right)_{1}$ are indices for $\lesssim_{a}$ in $2 F Q \forall$ and $2 F Q \exists$ forms respectively.

Proof. Let $i$ be a fixed index of the identity function and put $\theta^{\prime \prime}(a)$ $=\theta^{\prime}\left(3^{a} 5^{i}\right)$.

THEOREM 9. $\lesssim_{c}$ is expressible in both 2-function-quantifier forms. 
Proof. Let $\tilde{R}$ be the intersection of all binary relations $R$ on $N$ satisfying

(a) $D_{1} \subseteq R$,

(b) $x \in R \Rightarrow(\forall u)(\forall v)\left[\bar{Q}\left(3^{x} 5^{i}, u, v\right) \Rightarrow\langle u, v\rangle \in R\right]$,

(c) $x \in R \&(\forall u)\left[(\exists q) \bar{Q}\left(3^{x} 5^{i}, u, q\right) \Rightarrow \phi_{z}(u)\right.$ is defined \& $\left.(\exists q)\left(\left\langle\phi_{z}(u), q\right\rangle \in R\right)\right]$ $\&(\forall u)(\forall v)\left[\bar{Q}\left(3^{x} 5^{i}, u, v\right) \Leftrightarrow(\exists w)\left((\exists q) \bar{Q}\left(3^{x} 5^{i}, w, q\right) \& \bar{Q}\left(\phi_{z}(w), \phi_{z}(u), \phi_{z}(v)\right)\right)\right]$ $\Rightarrow(\forall u)(\forall v)\left[\bar{Q}\left(3^{x} 5^{z}, u, v\right) \Rightarrow\langle u, v\rangle \in R\right]$,

where $i$ is a fixed index of the identity function,

and

$$
\bar{Q}\left(y_{1}, y_{2}, y_{3}\right) \equiv(\forall \alpha)(\exists \beta)(\forall y) \sim T_{2}^{\alpha, \beta}\left(\left(\theta\left(y_{1}\right)\right)_{0}, y_{2}, y_{3}, y\right)
$$

$$
\overline{\overline{\mathrm{Q}}}\left(y_{1}, y_{2}, y_{3}\right) \equiv(\forall \alpha)(\exists \beta)(\forall y) \sim T_{2}^{\alpha, \beta}\left(\left(\theta^{\prime}\left(y_{1}\right)\right)_{0}, y_{2}, y_{3}, y\right) .
$$

We first show that $\widetilde{R}=\lesssim_{c}$. It is clear that both $\widetilde{R}$ and $\lesssim_{c}$ satisfy (a)-(c), so that $\tilde{R} \subseteq \lesssim_{c}$, $\left(\right.$ cf. $\left.\left(\mathrm{Id}_{2}\right)\right)$. Thus for $R=\tilde{R}$ the rules (a)-(c), reduce to

(a') $D_{1} \subseteq \tilde{R}$,

(b') $x \in \tilde{R} \Rightarrow D_{3^{2} \mathrm{~s}^{i}} \subseteq \tilde{R}$,

(c') $x \in \tilde{R} \& O P\left(z, D_{3^{x_{5}}}^{-}, \tilde{R}\right) \Rightarrow D_{3^{x_{5}}} \subseteq \tilde{R}$.

Suppose, now, that $\tilde{R} \neq \lesssim c$. Then there are integers $a \in C$ such that $a \notin \tilde{R}$; for otherwise $a \in C \Rightarrow a \in \widetilde{R} \Rightarrow D_{3^{a} b^{i}} \subseteq \widetilde{R}$, and hence $\lesssim_{C}=\bigcup_{a \in C} D_{3^{a} b^{i}} \subseteq \widetilde{R}$. So let $a$ be a minimal element of $C$ not in the domain of $\widetilde{R}$. Then $a \neq 1$ by $\left(a^{\prime}\right)$. If $a=2^{y}$, then $y \in \tilde{R}$, hence by $\left(\mathrm{b}^{\prime}\right), a=2^{y} \in D_{3^{y} 5^{i}} \subseteq \tilde{R}$ contrary to the choice of $a$. And if $a=3^{x} 5^{z}$, then $x \in \widetilde{R}$ so by $\left(\mathrm{b}^{\prime}\right), D_{3^{z} 5^{i}} \subseteq \widetilde{R}$. Now $O P C\left(z, D_{3^{z} 5^{i}}^{-}, D_{3^{x} z^{z}}^{-}\right)$and hence by choice of $a, O P\left(z, D_{3^{2} \varepsilon^{i}}^{-}, \widetilde{R}\right)$. But then by $\left(\mathrm{c}^{\prime}\right), D_{3^{z^{z}} \mathrm{~s}^{z}} \subseteq \tilde{R}$ contrary to the choice of $a$. Therefore $\widetilde{R}=\lesssim_{c}$.

The same result would be obtained if any or all of the occurrences of $\bar{Q}\left(y_{1}, y_{2}, y_{3}\right)$ were replaced by

$$
(\exists \alpha)(\forall \beta)(\exists y) T_{2}^{\alpha, \beta}\left(\left(\theta\left(y_{1}\right)\right)_{1}, y_{2}, y_{3}, y\right)
$$

and if any or all of the occurrences of $\bar{Q}\left(y_{1}, y_{2}, y_{3}\right)$ were replaced by

$$
(\exists \alpha)(\forall \beta)(\exists y) T_{2}^{\alpha, \beta}\left(\left(\theta^{\prime}\left(y_{1}\right)\right)_{1}, y_{2}, y_{3}, y\right) .
$$

Let $P$ be the predicate defined by

$$
\begin{aligned}
P(m, n) & \equiv(\forall \alpha) \cdot\{\alpha(1,1)=0 \&(\forall x)[(\exists q)(\alpha(x, q)=0) \\
& \left.\Rightarrow(\forall u)(\forall v)\left(Q_{1}\left(3^{x} 5^{i}, u, v\right) \Rightarrow \alpha(u, v)=0\right)\right] \&(\forall x)(\forall z)[(\exists q)[\alpha(x, q)=0] \\
& \&(\forall u)\left[(\exists q) Q_{2}\left(3^{x} 5^{i}, u, q\right) \Rightarrow \phi_{z}(u) \text { is defined \& }(\exists q)\left(\alpha\left(\phi_{z}(u), q\right)=0\right)\right] \\
& \&(\forall u)(\forall v)\left[Q _ { 2 } ( 3 ^ { x } 5 ^ { i } , u , v ) \Leftrightarrow ( \exists w ) \left((\exists q) Q_{2}\left(3^{x} 5^{i}, w, q\right)\right.\right. \\
& \left.\left.\left.\left.\& Q_{1}\left(\phi_{z}(w), \phi_{z}(u), \phi_{z}(v)\right)\right)\right] \Rightarrow(\forall u)(\forall v)\left[Q_{1}\left(3^{x} 5^{z}, u, v\right) \Rightarrow \alpha(u, v)=0\right]\right]\right\} \\
& \Rightarrow \alpha(m, n)=0,
\end{aligned}
$$

where $Q_{1}$ and $Q_{2}$ are predicate letters.

Applying Lemma 19 with $r=2, k=2$, 


$$
\begin{aligned}
& Q_{1}^{0}\left(y_{1}, y_{2}, y_{3}\right) \equiv(\forall \alpha)(\exists \beta)(\forall y) \sim T_{2}^{\alpha, \beta}\left(\left(\theta\left(y_{1}\right)\right)_{0}, y_{2}, y_{3}, y\right), \\
& Q_{1}^{1}\left(y_{1}, y_{2}, y_{3}\right) \equiv(\exists \alpha)(\forall \beta)(\exists y) T_{2}^{\alpha, \beta}\left(\left(\theta\left(y_{1}\right)\right)_{1}, y_{2}, y_{3}, y\right), \\
& Q_{2}^{0}\left(y_{1}, y_{2}, y_{3}\right) \equiv(\forall \alpha)(\exists \beta)(\forall y) \sim T_{2}^{\alpha, \beta}\left(\left(\theta^{\prime}\left(y_{1}\right)\right)_{0}, y_{2}, y_{3}, y\right),
\end{aligned}
$$

and

$$
Q_{2}^{1}\left(y_{1}, y_{2}, y_{3}\right) \equiv(\exists \alpha)(\forall \beta)(\exists y) T_{2}^{\alpha, \beta}\left(\left(\theta^{\prime}\left(y_{1}\right)\right)_{1}, y_{2}, y_{3}, y\right),
$$

we obtain predicates $P_{1}$ and $P_{2}$ such that

$$
P_{1}(m, n) \equiv(\forall \alpha)(\exists \beta)(\forall y) \sim T_{2}^{\alpha, \beta}\left(p_{1}, m, n, y\right)
$$

and

$$
P_{2}(m, n) \equiv(\exists \alpha)(\forall \beta)(\exists y) T_{2}^{\alpha, \beta}\left(p_{2}, m, n, y\right)
$$

for some fixed integers $p_{1}$ and $p_{2}$. Moreover by the above discussion of $\tilde{k}$,

$$
P_{1}(m, n) \equiv P_{2}(m, n) \equiv m \lesssim c n . \quad \text { Q.E.D. }
$$

COROLlaRY 1. $<_{c}$ is expressible in both 2-function-quantifier forms.

COROllary 2. $C$ is expressible in both 2-function-quantifier forms.

7. Extension of Kleene's $\mathbb{Q}$-hierarchy through $C$. The finite $\mathfrak{Q}$-hierarchy $\mathfrak{R}_{0}, \mathfrak{R}_{1}, \mathfrak{R}_{2}, \cdots$ of Kleene $\left[\mathrm{K}_{3}\right]$ is obtained by repeated application to a recursive predicate of the "hyperjump" operation

$$
R^{h}(a) \equiv(\forall \alpha)(\exists x) T_{1}^{R, \alpha}(a, a, x) .
$$

In [AK] Addison and Kleene show that the predicates in this hierarchy and in its extension (as their $\mathfrak{S}$-hierarchy) through the constructive third number class are expressible in both 2 -function-quantifier forms and hence, except for $\mathfrak{R}_{0}$ and $\mathfrak{R}_{1}$, are properly between $\mathfrak{N}_{1}$ and $\mathfrak{R}_{2}$ in hyperdegree $\left[\mathfrak{N}_{1}(a)\right.$ $\equiv(\forall \alpha)(\exists x) T_{1}^{\alpha}(a, a, x)$ and $\left.\mathfrak{N}_{2}(a) \equiv(\exists \alpha)(\forall \beta)(\exists x) T_{1}^{\alpha, \beta}(a, a, x)\right]$. This result is interesting for several reasons. First, it underlines the extent to which the hyperjump operation fails to play a role for the analytical hierarchy analogous to that played by Kleene's ordinary jump operation [KP] in the arithmetical hierarchy. Hence, second, it leaves open the problem of providing some such structure for the analytical hierarchy. And, third, it raises the question as to how far this hyperjump operation can be extended into the transfinite without reaching predicates which are not expressible in both 2-function-quantifier forms, or at least the problem of the extent to which this question can be made meaningful. 
In $\S 8$ we shall extend the $\mathfrak{S}$-hierarchy through our extension $\widetilde{C}$ of Addison and Kleene's constructive third number class and shall show that except for $\mathfrak{S}_{1}$ and $\mathfrak{W}_{2}$ this entire hierarchy still lies properly between $\mathfrak{R}_{1}$ and $\mathfrak{N}_{2}$ in hyperdegree. We are interested in this paragraph in obtaining analogous results for our system $C$ of notations.

Definition. For $a \in C, \Im_{a}$ is defined as follows: ( $i$ is a fixed index of the identity function)

$$
\Im_{a}(x) \equiv\left\{\begin{array}{lr}
x=x & \text { if } a=1, \\
(\forall \alpha)(\exists y) T_{1}^{\Im_{b}, \alpha}(x, x, y) & \text { if } a=2^{b}, \\
(x)_{1} \in{\overline{3^{b} b^{i}} \&}_{\Im_{\phi_{z}\left((x)_{1}\right)}}\left((x)_{0}\right) & \text { if } a=3^{b} 5^{z} .
\end{array}\right.
$$

Since $a<_{c} b$ implies that $\Im_{a}$ is of lower hyperdegree than $\Im_{b}$ (this can be proved by induction on $C$ ), and since $\Im_{2}=\Omega_{1}$, the fact that the predicates $\Im_{a}, 2<_{c} a$, are properly between $\mathfrak{N}_{1}$ and $\mathfrak{N}_{2}$ in hyperdegree follows from the next theorem.

THEOREM 10. There exists a recursive function $g$ such that for any $a \in C$, $(g(a))_{0}$ and $(g(a))_{1}$ are indices for $\Im_{a}$ in $2 F Q \forall$ and $2 F Q \exists$ forms respectively.

Proof. As in Theorem 8 the proof consists of an application of Lemma 20 to the well-ordered partial ordering $<_{c}$. The relation $\mathfrak{D}(x, S)$ of Lemma 20 is defined in this proof for an integer $x$ and segment $S$ of $C$ by:

$\mathcal{D}(x, S) \equiv\left\{\phi_{x}\right.$ is defined on $S$, and for each $b \in S,\left(\phi_{x}(b)\right)_{0}$ and $\left(\phi_{x}(b)\right)_{1}$ are indices for $\Im_{b}$ in $2 F Q \forall$ and $2 F Q \exists$ forms respectively .

The recursive function $f(x, a)$ is defined exactly as before except that $e_{1}$ and $e_{2}$ are changed. $e_{1}$ and $e_{2}$ are defined below.

CASE 1. $a=1$ : Let $e_{1}$ and $e_{2}$ define recursively from $\alpha$ and $\beta$ the functions

$$
\lambda x \mu y[x \neq x \& y=0]
$$

and

$$
\lambda x \mu y[x=x \& y=0]
$$

respectively.

CASE 2. $a=2^{b} \& b \neq 0$ : If $a \in C$,

$$
\begin{aligned}
\Im_{a}(x) & \equiv(\forall \alpha)(\exists y) T_{1}^{\Im_{b} \alpha^{\alpha}}(x, x, y) \\
& \equiv(\forall \alpha)(\exists y) T_{1}^{1,1}(\bar{\lambda}(y), \bar{\alpha}(y), x, x, y),
\end{aligned}
$$

where $\lambda$ is the representing function of $\Im_{b}$. Let $P(z, a, x)$ be the predicate obtained from

$$
(\forall \alpha)(\exists y)(\exists u)(\exists v)\left[u=\bar{\lambda}(y) \& v=\bar{\alpha}(y) \& T_{1}^{1,1}(u, v, x, x, y)\right]
$$

by replacing $u=\bar{\lambda}(y)$ by 
$(\exists w)\left\{(\forall i)\left[i<y . \Rightarrow .\left((w)_{i}=0 \& Q(z, b, i) . \vee \cdot(w)_{i}=1\right.\right.\right.$

$$
\left.\& \sim Q(z, b, i))] \&\left[\prod_{i<\nu} p_{i}^{(w)_{i}+1}=u\right]\right\} .
$$

Now, applying Lemma 19 with $r=1, k=2$,

$Q^{0}\left(y_{1}, y_{2}, y_{3}\right) \equiv \phi_{y_{1}}\left(y_{2}\right)$ is defined \& $(\forall \alpha)(\exists \beta)(\forall y) \sim T_{1}^{\alpha, \beta}\left(\left(\phi_{y_{1}}\left(y_{2}\right)\right)_{0}, y_{3}, y\right)$,

and

$$
Q^{1}\left(y_{1}, y_{2}, y_{3}\right) \equiv \phi_{y_{1}}\left(y_{2}\right) \text { is defined \& }(\exists \alpha)(\forall \beta)(\exists y) T_{1}^{\alpha, \beta}\left(\left(\phi_{y_{1}}\left(y_{2}\right)\right)_{1}, y_{3}, y\right),
$$

we obtain predicates $P_{1}$ and $P_{2}$ such that

$$
P_{1}(z, a, x) \equiv(\forall \alpha)(\exists \beta)(\forall y) \sim T_{3}^{\alpha, \beta}\left(p_{1}, z, a, x, y\right)
$$

and

$$
P_{2}(z, a, x) \equiv(\exists \alpha)(\forall \beta)(\exists y) T_{3}^{\alpha, \beta}\left(p_{2}, z, a, x, y\right)
$$

for some fixed integers $p_{1}$ and $p_{2}$. Let $e_{i}^{\prime}$ define recursively from $\alpha$ and $\beta$ the function

$$
\lambda p_{i} z a x \mu y T_{3}^{\alpha, \beta}\left(p_{i}, z, a, x, y\right)
$$

and let

$$
e_{i}=S_{1}^{3,1,1}\left(e_{i}^{\prime}, p_{i}, z, a\right), \quad i=1,2 .
$$

CAse 3. $a=3^{b} 5^{t}$ : Let $P^{\prime}$ be defined by $P^{\prime}(z, a, x) \equiv(\exists q) Q_{1}\left(b,(x)_{1}, q\right) \& \phi_{t}\left((x)_{1}\right)$ is defined \& $Q_{2}\left(z, \phi_{t}\left((x)_{1}\right),(x)_{0}\right)$, and let $P_{1}^{\prime}$ and $P_{2}^{\prime}$ be obtained by means of the corollary to Lemma 19 with $r=2, k=2$,

$$
\begin{aligned}
& Q_{1}^{0}\left(y_{1}, y_{2}, y_{3}\right) \equiv(\forall \alpha)(\exists \beta)(\forall y) \sim T_{2}^{\alpha, \beta}\left(\left(\theta^{\prime \prime}\left(y_{1}\right)\right)_{0}, y_{2}, y_{3}, y\right), \\
& Q_{1}^{1}\left(y_{1}, y_{2}, y_{3}\right) \equiv(\exists \alpha)(\forall \beta)(\exists y) T_{2}^{\alpha, \beta}\left(\left(\theta^{\prime \prime}\left(y_{1}\right)\right)_{1}, y_{2}, y_{3}, y\right), \\
& Q_{2}^{0}=Q^{0} \text { of Case 2, }
\end{aligned}
$$

and

$$
Q_{2}^{1}=Q^{1} \text { of Case } 2
$$

such that 


$$
P_{1}^{\prime}(z, a, x) \equiv(\forall \alpha)(\exists \beta)(\forall y) \sim T_{3}^{\alpha, \beta}\left(p_{1}^{\prime}, z, a, x, y\right)
$$

and

$$
P_{2}^{\prime}(z, a, x) \equiv(\exists \alpha)(\forall \beta)(\exists y) T_{3}^{\alpha, \beta}\left(p_{2}^{\prime}, z, a, x, y\right)
$$

for some fixed integers $p_{1}^{\prime}$ and $p_{2}^{\prime}$. Let $e_{i}^{\prime \prime}$ define recursively from $\alpha$ and $\beta$ the function

$$
\lambda p_{i} z a x \mu y T_{3}^{\alpha, \beta}\left(p_{i}^{\prime}, z, a, x, y\right),
$$

and let

$$
e_{i}=S_{1}^{3,1,1}\left(e_{i}^{\prime \prime}, p_{i}^{\prime}, z, a\right), \quad i=1,2 .
$$

Case 4. Otherwise let $e_{1}=e_{2}=0$.

The proof is completed as in Theorem 8 by showing that condition (iii) of Lemma 20 is satisfied; i.e. as before it is only necessary to show that for $a \in C$ and $z$ such that $\mathfrak{D}\left(z, S_{a}\right), e_{1}$ and $e_{2}$ are indices in both 2-function-quantifier forms for $\Im_{a}$.

If $a=1$ it is clear that $e_{1}$ and $e_{2}$ are indices for $\Im_{1}$ as required.

If $a=2^{b} \& b \neq 0$ then $e_{1}$ and $e_{2}$ are indices for $\lambda x P_{1}(z, a, x)$ and $\lambda x P_{2}(z, a, x)$ in $2 F Q \forall$ and $2 F Q \exists$ forms respectively. By hypothesis, $\lambda i Q^{0}(z, b, i) \equiv \lambda i Q^{1}(z, b, i)$ $\equiv \Im_{b}(i)$; hence the expression

$$
\begin{array}{r}
(\exists w)\left\{(\forall i)\left[i<y . \Rightarrow \cdot\left((w)_{i}=0 \& Q(z, b, i) \cdot \vee \cdot(w)_{i}=1 \& \sim Q(z, b, i)\right)\right]\right. \\
\left.\&\left[\prod_{i<\nu} p_{i}^{(w)_{i}+1}=u\right]\right\},
\end{array}
$$

where $Q$ is either $Q^{0}$ or $Q^{1}$, is simply $\bar{\lambda}(y)=u$, where $\lambda$ is the representing function of $\Im_{b}$. Thus $\lambda x P_{1}(z, a, x) \equiv \lambda x P_{2}(z, a, x)$ $\equiv(\forall \alpha)(\exists y) T_{11}^{1,1}(\bar{\lambda}(y), \bar{\alpha}(y), x, x, y) \equiv \Im_{a}(x)$, as desired.

If $a=3^{b} 5^{t}$, then $e_{1}$ and $e_{2}$ are indices for $\lambda x P_{1}^{\prime}(z, a, x)$ and $\lambda x P_{2}^{\prime}(z, a, x)$ in $2 F Q \forall$ and $2 F Q \exists$ forms respectively. By hypothesis, $Q_{1}^{0}\left(b,(x)_{1}, q\right) \equiv Q_{1}^{1}\left(b,(x)_{1}, q\right)$ $\equiv\left\langle(x)_{1}, q\right\rangle \in D_{3^{b_{b}}}^{-}$, and hence

$$
\begin{aligned}
\lambda x P_{1}^{\prime}(z, a, x) & \equiv \lambda x P_{2}^{\prime}(z, a, x) \\
& \equiv(\exists q)\left[\left\langle(x)_{1}, q\right\rangle \in \overline{D_{s^{b} b^{i}}}\right] \& \Im_{\phi_{t}\left((x)_{1}\right)}\left((x)_{0}\right) \\
& \equiv \Im_{a}(x),
\end{aligned}
$$

showing that $e_{1}$ and $e_{2}$ have the desired properties. Q.E.D.

8. $2 F Q \forall$ and $2 F Q \exists$ forms for $\tilde{C}$. In this section we outline results for $\tilde{C}$ and $<_{\tilde{c}}$ corresponding to those obtained in $\$ \$ 6$ and 7 for $C$ and $<c$. Most of the details are omitted from the proofs in this section because of their similarity to preceding proofs. Complete proofs are found in $[\mathrm{Kr}]$. 
THEOREM 11. There exists a recursive function $\bar{\theta}$ such that for any $a \in \tilde{C}$,

$$
\begin{aligned}
\langle m, n\rangle \in I_{a} & \equiv(\forall \alpha)(\exists \beta)(\forall y) \sim T_{2}^{\alpha, \beta}\left((\bar{\theta}(a))_{0}, m, n, y\right) \\
& \equiv(\exists \alpha)(\forall \beta)(\exists y) T_{2}^{\alpha, \beta}\left((\bar{\theta}(a))_{1}, m, n, y\right) .
\end{aligned}
$$

Proof. As in Theorem 8 the proof consists of an application of Lemma 20 to the well-ordered partial ordering $<\tau$. The relation $\mathfrak{D}(x, S)$ of Lemma 20 is defined in this proof, for an integer $x$ and a segment $S$ of $\tilde{C}$, by

$\mathfrak{D}(x, S) \equiv\left\{\phi_{x}\right.$ is defined on $S$, and for each $b \in S,\left(\phi_{x}(b)\right)_{0}$ and $\left(\phi_{x}(b)\right)_{1}$ are indices for $I_{b}$ in $2 F Q \forall$ and $2 F Q \exists$ forms respectively .

In contrast to Theorem 8 where $\theta(a)$ gave $2 F Q$ forms for the segment of notations through $a$ (" $D_{a}$ "), $\bar{\theta}(a)$ in this theorem gives the $2 F Q$ forms for the number class of index $a$ (" $I_{a}$ ").

The recursive function $f(x, a)$ is again defined as in Theorem 8 except that $e_{1}, e_{2}$ are defined as below:

CASE 1. $a=1$ : Let $e_{1}, e_{2}$ define recursively from $\alpha, \beta$ the functions

$$
\lambda m n \mu y\left[\sim\left(\langle m, n\rangle \in I_{1}\right) \& y=0\right]
$$

and

$$
\lambda m n \mu y\left[\langle m, n\rangle \in I_{1} \& y=0\right]
$$

respectively.

CASE 2. $a=2^{b}$ and $b \neq 0$ : Let $P$ be the predicate defined by

$$
\begin{gathered}
P(x, a, m, n) \equiv(\forall \alpha) \cdot\left\{\alpha(1,2)=0 \&(\forall u)\left[\alpha(1, u)=0 \Rightarrow \alpha\left(u, 2^{u}\right)=0\right]\right. \\
\&(\forall u)(\forall v)(\forall w)[\alpha(u, v)=0 \& \alpha(v, w)=0 \Rightarrow \alpha(u, w)=0] \\
\&(\forall y)(\forall z)\left[[y=b \vee Q(x, b, y, b)] \&(\forall s)\left[(\exists q) Q(x, y, s, q) \Rightarrow \phi_{z}(s) \text { is defined }\right]\right. \\
\&(\forall s)(\forall t)\left[Q(x, y, s, t) \Rightarrow \alpha\left(\phi_{z}(s), \phi_{z}(t)\right)=0\right] \\
\&(\exists r)\left[(\exists q) Q(x, y, r, q) \& \alpha\left(y, \phi_{z}(r)\right)=0\right] \Rightarrow(\forall r)[(\exists q) Q(x, y, r, q) \\
\left.\left.\left.\Rightarrow \alpha\left(\phi_{z}(r), 3^{y} 5^{z}\right)=0\right]\right]\right\} \Rightarrow \alpha(m, n)=0 .
\end{gathered}
$$

(For motivation, see the definition of $\mathfrak{C}(A, R)$ and (2) of the definition of $\mathbb{R}$ in \$5.)

Applying Lemma 19 with $r=1, k=2$, and $Q^{0}$ and $Q^{1}$ as in the proof of Theorem 8 , we obtain predicates $P_{1}$ and $P_{2}$ such that

$$
P_{1}(x, a, m, n) \equiv(\forall \alpha)(\exists \beta)(\forall y) \sim T_{4}^{\alpha, \beta}\left(p_{1}, x, a, m, n, y\right)
$$

and

$$
P_{2}(x, a, m, n) \equiv(\exists \alpha)(\forall \beta)(\exists y) T_{4}^{\alpha, \beta}\left(p_{2}, x, a, m, n, y\right)
$$


for some fixed integers $p_{1}$ and $p_{2}$. Let $e_{i}^{\prime}$ define recursively from $\alpha, \beta$ the function

and let

$$
\lambda p_{i} x a m n \mu y T_{4}^{\alpha, \beta}\left(p_{i}, x, a, m, n, y\right),
$$

$$
e_{i}=S_{2}^{3,1,1}\left(e_{i}^{\prime}, p_{i}, x, a\right) \quad(i=1,2) .
$$

CASE 3. $a=3^{b} 5^{t}$ : Let $P^{\prime}$ be the predicate defined by $P^{\prime}(x, a, m, n) \equiv(\forall \alpha) \cdot\{\alpha(1,2)=0$

$$
\begin{aligned}
& . \& .(\forall u)\left[\alpha(1, u)=0 \Rightarrow \alpha\left(u, 2^{u}\right)=0\right] \\
& . \& .(\forall u)(\forall v)(\forall w)[\alpha(u, v)=0 \& \alpha(v, w)=0 \Rightarrow \alpha(u, w)=0] \\
& . \& .(\forall y)(\forall z)\left[( \exists v ) \left[(\exists q) Q(x, b, v, q) \& \phi_{t}(v)\right.\right. \text { is defined } \\
& \left.\qquad\left(y=\phi_{t}(v) \vee Q\left(x, \phi_{t}(v), y, \phi_{t}(v)\right)\right)\right] \\
& \text { \& }(\forall r)\left[(\exists q) Q(x, y, r, q) \Rightarrow \phi_{z}(r) \text { is defined }\right] \\
& \&(\forall r)(\forall s)\left[Q(x, y, r, s) \Rightarrow \alpha\left(\phi_{z}(r), \phi_{z}(s)\right)=0\right] \\
& \&(\exists r)\left[(\exists q) Q(x, y, r, q) \& \alpha\left(y, \phi_{z}(r)\right)=0\right] \\
& \left.\left.\Rightarrow(\forall r)\left[(\exists q) Q(x, y, r, q) \Rightarrow \alpha\left(\phi_{z}(r), 3^{y} 5^{z}\right)=0\right]\right]\right\} \\
& \Rightarrow \alpha(m, n)=0 .
\end{aligned}
$$

(For motivation: see (3) of the definition of $R$ in $\$ 5$.)

We now apply Lemma 19 as in Case 2(12).

CASE 4. Otherwise let $e_{1}=e_{2}=0$.

The proof is completed as in Theorem 8 by showing that condition (iii) of Lemma 20 is satisfied. This follows easily from the definition of $\tilde{C}$ in $\$ 5$. Q.E.D.

THEOREM 12. $<\tau$ is expressible in both 2-function-quantifier forms.

Proof. Define the predicate $P$ by

$$
\begin{gathered}
P(m, n) \equiv(\forall \alpha) \cdot\{(\forall u)(\forall v)[Q(1, u, v) \Rightarrow \alpha(u, v)=0] \\
\&(\forall x)[(\exists q)(\alpha(x, q)=0) \Rightarrow(\forall u)(\forall v)(Q(x, u, v) \Rightarrow \alpha(u, v)=0)] \\
\&(\forall x)(\forall z)\left[(\exists q)[\alpha(x, q)=0] \&(\forall u)\left[(\exists q) Q(x, u, q) \Rightarrow \phi_{z}(u) \text { is defined }\right]\right. \\
\&(\forall u)(\forall v)\left[Q(x, u, v) \Rightarrow \alpha\left(\phi_{z}(u), \phi_{z}(v)\right)=0\right] \\
\left.\left.\&(\exists u)\left[(\exists q) Q(x, u, q) \& \alpha\left(x, \phi_{z}(v)\right)=0\right] \Rightarrow(\exists q)\left[\alpha\left(3^{x} 5^{z}, q\right)=0\right]\right]\right\} \\
\left.\Rightarrow \alpha(m, n)=0 .{ }^{13}\right)
\end{gathered}
$$

(12) In Case 3, application of Lemma 20 depends upon the fact that the forms for $I_{b}$ will be obtained prior to $3^{b} 5^{t}$. Thus we use, here also, the regularity condition mentioned in (b) at the beginning of $\$ 5$. (See footnote 8.)

(13) That part of the definition of $P(m, n)$ which begins $(\forall x)(\forall z) \cdots$ is necessary in view of difficulty (a) discussed early in $\$ 5$. 
Applying Lemma 19 with $r=1, k=2$,

$$
Q^{0}\left(y_{1}, y_{2}, y_{3}\right) \equiv(\forall \alpha)(\exists \beta)(\forall y) \sim T_{2}^{\alpha, \beta}\left(\left(\bar{\theta}\left(y_{1}\right)\right)_{0}, y_{2}, y_{3}, y\right),
$$

and

$$
Q^{1}\left(y_{1}, y_{2}, y_{3}\right) \equiv(\exists \alpha)(\forall \beta)(\exists y) T_{2}^{\alpha, \beta}\left(\left(\bar{\theta}\left(y_{1}\right)\right)_{1}, y_{2}, y_{3}, y\right),
$$

we obtain predicates $P_{1}$ and $P_{2}$ such that

$$
P_{1}(m, n) \equiv(\forall \alpha)(\exists \beta)(\forall y) \sim T_{2}^{\alpha, \beta}\left(p_{1}, m, n, y\right)
$$

and

$$
P_{2}(m, n) \equiv(\exists \alpha)(\forall \beta)(\exists y) T_{2}^{\alpha, \beta}\left(p_{2}, m, n, y\right)
$$

for some fixed integers $p_{1}$ and $p_{2}$.

By methods similar to those of Theorem 9 we can now show that $P_{1}(m, n)$ $\equiv P_{2}(m, n) \equiv m<\tilde{c} n$, using Lemma 17 and other results from §5. Q.E.D.

COROLlaRY. $\tilde{C}$ is expressible in both 2-function-quantifier forms.

Consider now the Kleene $\mathfrak{R}_{k}$-hierarchy ( $k$ a natural number) extended through $\tilde{C}$ as the $\mathfrak{S}_{a}$-hierarchy (for $a \in \tilde{C}$ ) [AK]. We shall show, just as for the $\mathfrak{S}_{a}$-hierarchy $(a \in C)$, that for $2<\tilde{c} a, \mathfrak{S}_{a}$ is properly between $\mathfrak{N}_{1}$ and $\mathfrak{N}_{2}$ in hyperdegree.

Definition. For $a \in \tilde{C}, \mathfrak{S}_{a}$ is defined as follows:

$$
\mathfrak{S}_{a}(x) \equiv\left\{\begin{array}{lr}
x=x & \text { if } a=1 \\
(\forall \alpha)(\exists y) T_{1}^{\mathfrak{W}_{b}, \alpha}(x, x, y) & \text { if } a=2^{b} \\
(x)_{1} \in I_{b} \& \mathfrak{E}_{\phi_{z}\left((x)_{1}\right)}\left((x)_{0}\right) & \text { if } a=3^{b} 5^{z}
\end{array}\right.
$$

As in the case of the $\Im_{a}$-hierarchy, $\mathfrak{S}_{2}$ is of maximal hyperdegree for 1function-quantifier predicates, $\mathfrak{S}_{2^{b}}(b \in \tilde{C})$ is of greater hyperdegree than $\mathfrak{S}_{b}$, and $\mathfrak{S}_{3 b^{z}}\left(3^{b} 5^{z} \in \tilde{C}\right)$ is of greater hyperdegree than $\mathfrak{S}_{\phi_{z}(v)}$ for any $v \in I_{b}$. Hence to show that the $\mathfrak{W}_{a}$-hierarchy (after $\mathfrak{S}_{1}, \mathfrak{S}_{2}$ ) lies properly between $\mathfrak{N}_{1}$ and $\mathfrak{R}_{2}$ in hyperdegree, it is sufficient to show that $\mathfrak{S}_{a}(a \in \widetilde{C})$ is expressible in both 2-function-quantifier forms.

THEOREM 13. There exists a recursive function $\bar{g}$ such that for any $a \in \tilde{C}$,

$$
\begin{aligned}
\mathfrak{S}_{a}(x) & \equiv(\forall \alpha)(\exists \beta)(\forall y) \sim T_{1}^{\alpha, \beta}\left((\bar{g}(a))_{0}, x, y\right) \\
& \equiv(\exists \alpha)(\forall \beta)(\exists y) T_{1}^{\alpha, \beta}\left((\bar{g}(a))_{1}, x, y\right) .
\end{aligned}
$$

Proof. The proof is the same as the proof of Theorem 10 , reading $\mathfrak{S}_{a}$ for $\Im_{a}, \mathfrak{S}_{b}$ for $\Im_{b}, \bar{\theta}$ for $\theta^{\prime \prime}, \tilde{C}$ for $C$, and $\bar{g}$ for $g$. Q.E.D. 


\section{Concluding discussion.}

Other literature. Systems of notation extending beyond the constructive ordinals have been considered by Church and Kleene in [CK] and by Wang in $\left[\mathrm{W}_{1} ; \mathrm{W}_{2}\right]$. As mentioned in $\$ 2$ above, the system $S_{1}$ for the constructive ordinals is trivially isomorphic to the main system of [CK]; see comments in $\left[\mathrm{K}_{1}, \S 3\right]$. Our system $C$ is isomorphic to the extended system of [CK]. The structure of $<_{c}$ as a linear ordering of equivalence classes (see after Theorem 4 in $\$ 4$ above) is given in [CK] by the concept of "formally defined function of ordinals" on p. 14 of [CK]. $\left(1^{*}\right)$ of $\$ 4$ above is given by rule (iii) of [CK] (with $S_{0}(a)$ in place of the function $\left.2^{a}\right) .\left(2^{*}\right)$ and $\left(3^{*}\right)$ of $\$ 4$ above are given by rules (iva) of $[\mathrm{CK}]$ (with $\left(3^{*}\right)$ corresponding to the special case $b=\iota_{a}$, and with $L(a, r)$ in place of $\left.3^{a} 5^{r}\right)$.

The present authors are indebted to Wang. They were in part stimulated to their present study by the valuable insights imparted by him at the Cornell Summer Institute in 1957. At the same time, in view of the small amount of existing literature, they feel impelled to indicate that some of the written material of $\left[\mathrm{W}_{1} ; \mathrm{W}_{2}\right]$ is unclear and that some is erroneous. We comment as follows:

(1) In both papers systems are described which are said to embody the extended system of [CK]. However, the systems described can be seen to be of the $S_{3}$-type rather than of the $S_{1}$-type of [CK].

(2) The system of $\left[W_{1}\right]$ does not meet difficulty (a) of $\$ 5$ above and hence does not carry beyond the constructive counterpart to $\omega_{\omega_{\omega}} \ldots$. It does not, furthermore, use the regularity condition of (b) of $\$ 5$, which appears necessary for the full extension of $S_{3}$.

(3) As extensions of $S_{3}$, these systems of $\left[\mathrm{W}_{1} ; \mathrm{W}_{2}\right]$ are subject to the various complexity difficulties described in $\$ 3$ above.

(4) The system of $\left[W_{1}\right]$ is not a simple extension in the usual sense, for with each addition of a constructive number class, new notations are given to all ordinals back to $\omega$ in the 2 nd constructive number class (via the factor $7^{a}$, see $\left[W_{1}\right.$, p. 385]).

(5) In the general discussion of "systems of notation" in $\left[\mathrm{W}_{2}\right]$, the treatment of finite ordinals is left unclear, and the limitation to univalent (one notation for each ordinal) systems in $\left[\mathrm{W}_{2}\right.$, Definition 2] seems unintended and probably a typographical error. Also in this general discussion, Definition $4 u$ fails of its intent. The intersection defined gives only finite notations. The evident correction, i.e. deletion of " $V\left(1,3^{f}\right)$ ", is not entirely satisfactory since it is by no means clear that the corrected system would cover all constructive ordinals, as is asserted without proof (for the uncorrected system).

(6) The particular discussion in $\left[\mathrm{W}_{2}\right]$ of the extended system of $[\mathrm{CK}]$ has several further difficulties, in addition to (1) and (3) above. The informal restatement of the Church-Kleene "rules" is inadequate and does not lead beyond the 2 nd constructive number class. The evident correction here (from 
" $\gamma_{1}<\alpha$ " to " $\left.\gamma_{1} \leqq \alpha "\right)$ is still not adequate, since the resulting rules do not handle the case of those notations $n$ which appear for the first time in the number of class of index $n$. (See difficulties (a) discussed in $\$ 3$ above. The original Church-Kleene rules are adequate in these respects.) The formal system $B$ (and hence $C$ ) of $\left[\mathrm{W}_{2}\right]$ also suffers from the problem last mentioned. See the occurrences of " $u$ " in 3.7.4 of $\left[\mathrm{W}_{2}\right]$. This may lead to intersection difficulties of the kind considered at the end of $\$ 3$ above. Implementation of the suggestions attributed to the present authors in $\left[\mathrm{W}_{2}\right]$ appears to be not wholly successful.

Alternative formulations. As we noted in $\$ 3$, useful features of $S_{3}$ are lost in extensions like $C$ and $\tilde{C}$. The looser effective structure of such extensions, however, gives freedom to make nonconstructive modifications, by "fiat". We mention several of many possible modifications of $C$. One of these is the system suggested to the writers by Davis and Putnam and called $C^{*}$ in [DP]. In $C^{*}$, notations introduced by $\left(3^{*}\right)$ are distinguished from those introduced by $\left(2^{*}\right)$. (I.e. the "type numbers" (see $\$ 1$ ) are given distinctive notations.) In one form of $C^{*}$, - to give a rough description-, the modified $\left(3^{*}\right)$ would introduce notations $3^{3^{b}} 5^{e}$ where the present $\left(3^{*}\right)$ introduces $3^{b} 5^{e}$. Another possible modification of $C$ would be to label notations-as far as possible-according to the constructive number class in which they first occur. This may be the intended purpose of the factor $7^{a}$ in the systems of $\left[W_{1} ; W_{2}\right]$. We have not made such modifications, but have chosen to remain with an extension directly isomorphic to that of [CK].

Problems of equivalence. The question of containment between two systems can be asked in a weak form: does one system cover as large a segment of ordinals as the other? It can be asked in a strong form: is there an effective mapping into one system from the other? The questions of containment, in either sense and in either direction, between $C$ and $\tilde{C}$ remain open and appear difficult. (The only methods known for handling such questions are those which would give the stronger containment by an application of the Recursion Theorem (Lemma 20).)

In [DP], Davis and Putnam announce a solution of the uniqueness problem for the jump operation iterated through $C^{*}$, (see [S]). They also announce the formulation of a concept of "extended $R$-system" generalizing the concept of " $r$-system" in $\left[\mathrm{K}_{1}\right]$, and that $C^{*}$ is a maximal extended $R$-system. A proof of this latter result, giving strong containment via the Recursion Theorem as in Lemma 20, must overcome the following difficulty. Let $\bar{C}$ be any extended $R$-system. Let $\varphi$ be the mapping being constructed. In general, $\varphi$ will have mapped a constructive number class $\bar{I}$ (of index $b$ ) of $\bar{C}$ properly into (as sets of integers) a corresponding constructive number class $I^{*}$ (of index $\varphi(b))$ of $C^{*}$. But now the image under $\varphi$ of $3^{b} 5^{e}$ will be $3^{\varphi(b)} 5^{e^{\prime}}$ where $e^{\prime}$ is a Gödel number for $\varphi \phi_{e} \varphi^{-1}$. However since $\varphi$ maps $\bar{I}$ properly into $I^{*}, \phi_{\varepsilon}$ will not be defined for all of $I^{*}$ as is required for $3^{\varphi(b)} 5^{e^{\prime}}$ to be a notation. 
Open questions. In addition to the problems of containment and equivalence between $C$ and $\tilde{C}$, certain other questions remain open.

(a) Does the analogue of Result 10 in $\$ 1$ hold for $C$ (or $\tilde{C}$ ) ? I.e., if $\left|3^{b_{1} 5^{e_{1}}}\right|_{c}=\left|3^{b_{2}} 5^{e_{2}}\right| c$, does it follow that $\left|b_{1}\right|_{c}=\left|b_{2}\right|_{c}$ ? Proof of the classical result has nonconstructive features that do not directly carry over.

(b) The systems $S_{1}$ and $S_{3}$ can also be extended by allowing sequences to be relative recursive in a given set. See the system $O^{\circ}$ in $\left[\mathrm{K}_{3}\right]$. How do such systems compare with $C$ (or $\tilde{C}$ ) as to the segment of ordinals covered?

(c) Can a theory for some system like $C$ (or $\tilde{C}$ ) be developed which will include a recursive analogue to parts of classical cardinal number theory?

(d) Can the regularity condition for $\tilde{C}$ (see (b) in \$5) be omitted or proved?

Further extensions. In the Final Comment of $\$ 1$, certain additional principles for generating segments of the classical ordinals are mentioned. These principles can be paralleled by further extensions of $C$. (It will be noted that such extensions go more easily with $C$ than with $\tilde{C}$.) Thus,

(i) at the " $\alpha$ th point of 2 nd order difficulty", we could introduce the notation $3^{a} 5^{e} 7^{4}$, where $a$ is a notation for $\alpha$ and $e$ maps the preceding segment into itself;

(ii) at the " $\alpha$ th point of $\beta$ th order difficulty", we could use $3^{a} 5^{e} 7^{b}$, where $a$ and $b$ are notations for $\alpha$ and $\beta$, and where $e$ is as before;

(iii) at the " $\alpha$ th point of $\gamma$ th order super difficulty", we could use $3^{a} 5^{e} 11^{c}$, etc., etc. Clearly such extensions can be carried far beyond those indicated. Their existence supports the view that the "constructively accessible" ordinals covered by $C$ may not give as natural a stopping place as the "constructively countable" ordinals covered by $S_{1}$.

This suggests several interesting goals for further research. One of these is to find, in some appropriate weak sense, a quasi-constructive system of notations that includes all extensions of the kind just indicated. A further goal would be to get a system that would be in complete $2 F Q \forall$ form and that would carry the hyperjump (or some stronger version thereof) from $\mathfrak{N}_{1}$ through $\mathfrak{R}_{2}$ (see $\S 7$ above). A related area of research would be a study of the possible metamathematical role of such systems in extensions of set theory of the kind considered by Lévy in [L].

\section{BIBLIOGRAPHY}

[AK] J. W. Addison and S. C. Kleene, A note on function quantification, Proc. Amer. Math. Soc. vol. 8 (1957) pp. 1002-1006. 1955.

[B] H. Bachmann, Transfinite Zahlen, Berlin, Göttingen, Heidelberg, Springer-Verlag,

[C] A. Church, The constructive second number class, Bull. Amer. Math. Soc. vol. 44 (1938) pp. 224-232.

[CK] A. Church and S. C. Kleene, Formal definitions in the theory of ordinal numbers, Fund. Math. vol. 28 (1937) pp. 11-21. 
[DP] H. Putnam and M. Davis, Uniqueness ordinals and extended R-systems, Abstract 549-30, Notices Amer. Math. Soc. vol. 5 (1958) p. 607.

[F] A. A. Fraenkel, Abstract set theory, Amsterdam, North Holland, 1953.

[G] K. Gödel, The consistency of the continuum hypothesis, Princeton, Princeton University Press, 1940. 155.

[K $\left.\mathrm{K}_{1}\right] \mathrm{S} . \mathrm{C}$. Kleene, On notation for ordinal numbers, J. Symb. Logic vol. 3 (1938) pp. 150-

$\left[\mathrm{K}_{2}\right] \longrightarrow$, Arithmetical predicates and function quantifiers, Trans. Amer. Math. Soc. vol. 79 (1955) pp. 312-340.

$\left[\mathrm{K}_{3}\right] \longrightarrow$, Hierarchies of number-theoretic predicates, Bull. Amer. Math. Soc. vol. 61 (1955) pp. 193-213.

$\left[\mathrm{K}_{4}\right] \longrightarrow$, Introduction to metamathematics, New York and Toronto, Van Nostrand; Amsterdam, North Holland; Groningen, Noordhoff; 1952.

[KP] S. C. Kleene and E. L. Post, The upper semi-lattice of degrees of recursive unsolvability, Ann. of Math. vol. 59 (1954) pp. 379-407.

[Kr] D. L. Kreider, Analytic predicates and extensions of the notion of constructive ordinal, $\mathrm{Ph} . \mathrm{D}$. thesis, Massachusetts Institute of Technology, 1959.

[L] A. Lévy, Axiom schemata of strong infinity in axiomatic set theory, Pacific J. Math. vol. 10 (1960) pp. 223-238.

[R] H. Rogers, Jr., Recursive functions over well-ordered partial orderings, Proc. Amer. Math. Soc. vol. 10 (1959) pp. 847-853.

[S] C. Spector, Recursive well-orderings, J. Symb. Logic vol. 20 (1955) pp. 151-163.

[V] O. Veblen, Continuous increasing functions of finite and transfinite ordinals, Trans. Amer. Math. Soc. vol. 9 (1908) pp. 280-292.

$\left[\mathrm{W}_{1}\right] \mathrm{H}$. Wang, Remarks on constructive ordinals and set theory, Proc. Summer Institute of Symbolic Logic at Cornell University vol. 3 (1957) pp. 383-390.

$\left[\mathrm{W}_{2}\right] \longrightarrow$, Ordinal numbers and predicative set theory, $Z$. Math. Logik Grundlagen Math. vol. 5 (1959) pp. 216-239.

Massachusetts Institute of Technology, Cambridge, Massachusetts 